\title{
DELAY IN A TANDEM QUEUEING MODEL WITH MOBILE QUEUES: AN ANALYTICAL APPROXIMATION
}

\author{
Ahmad al Hanbali \\ Department of Industrial Engineering and Business Information Systems, IEBIS Group \\ School of Management and Governance \\ University of Twente \\ The Netherlands \\ E-mail: a.alhanbali@utwente.nl
}

\author{
Roland de HaAn, Richard J. Boucherie and Jan-Kees van Ommeren \\ Dep. of Applied Mathematics, SOR Group \\ University of Twente \\ The Netherlands \\ E-mail: haanr@utwente.nl, r.j.boucherie@utwente.nl, j.c.w.vanommeren@utwente.nl
}

\begin{abstract}
In this paper, we analyze the end-to-end delay performance of a tandem queueing system with mobile queues. Due to state-space explosion, there is no hope for a numerical exact analysis for the joint-queue-length distribution. For this reason, we present an analytical approximation that is based on queue-length analysis. Through extensive numerical validation, we find that the queue-length approximation exhibits excellent performance for light traffic load.
\end{abstract}

\section{INTRODUCTION}

The model considered in this paper, originated from the study of ad hoc networks, in which the end-to-end connectivity is not always guaranteed. For instance, nodes in the network may vary their transmission power, they may move, they may enter the sleep mode, or they may suffer from hardware failures. As a result, the network structure changes dynamically and this may lead to undesired situations of nodes becoming disconnected from parts of the network. The traditional store-and-forward routing protocols cannot be employed in highly disconnected ad hoc networks. A solution for this problem is to exploit the mobility of nodes present in the network. Such an approach has been proposed in the pioneering paper of Grossglauser and Tse [12] as an alternative to the store-and-forward paradigm and it is now known as the store-carry-and-forward paradigm in the context of delaytolerant networking [8]. As a first step toward the analysis of this system, we consider a very simple scenario with a one-way stream of messages over a fixed route of relay nodes with a restricted mobility. We model this situation as a tandem-queueing model where each queue randomly switches between two positions. We focus on approximations for the end-to-end delay performance. 
The network model of our interest is reminiscent of a multi-queue tandem model with multiple alternating servers, which move among the queues autonomously. In the literature, it is usually assumed that the server can be controlled. Tandem models with controlled servers have been analyzed under various servicing strategies in the special case of a single server (see, e.g., [18]). In a two-queue setting, [5] analyzes the model via boundary value techniques. Unfortunately, the analysis along these lines for more than two queues appears intractable. Time-limited service models with server control have also been studied in the context of polling systems (see, e.g., $[10,11,13]$ ), where the server moves to another queue when it becomes empty. In the mobility-driven model of our interest, the server is autonomous and there is no possibility to control its movement.

An important step toward understanding the impact of mobility on the end-to-end delay, is the study in [1] of a model comprising a fixed source and destination queue, and a single mobile queue operating as a relaying device. Modeling this network as a tandem of queues, we performed an exact analysis for the joint queue length by extending the techniques developed in $[2,7,13]$. Due to the state-space explosion, the computation time of the joint queue-length probabilities may grow large for certain model parameters. Therefore, as a complementary tool, we presented an analytical approximation for the case that the service requirements at each queue are exponential. In this paper, we are interested in the model comprising multiple mobile queues. Unfortunately, the exact analysis carried out in [1] is numerically intractable in this model due to the increase in the number of queues. For this reason, we will focus on the approximation. As a generalization, we will allow the distribution of the service requirements at the different queues to be general.

Our main interest is in the end-to-end delay in the network described above. The main complexity in our model is the correlation between the queue lengths at different queues. A numerically efficient approximation will be presented. The main idea is to relate the sojourn time at a mobile queue and its queue-length process at specific embedded epochs. The queuelength process at these embedded epochs is then analyzed in isolation as a discrete-time queue with geometric batch arrivals. The key element is to approximate the batch arrival process with correlated batch sizes with a batch process of independent batch size. This approximation is referred to as queue-length approximation.

Note that the arrivals to a queue are the departures of the upstream queue in the tandem. Therefore, to derive the queue length of queue $i$, it is required to first analyze queue $i-1$, and so on. Thus, our approximation is based on an iterative scheme that derives the delay at queue one first, then at queue two, and so on. A similar iterative scheme was used recently in [17] for the analysis of multi-server tandem queues with finite buffers and blocking.

The rest of the paper is organized as follows. Section 2 presents our model. The stability condition of the system is derived in Section 3. In Section 4, we present exact results for the sojourn time in the source queue. Section 5 proposes and analyzes the approximation for the sojourn time in the mobile queue via queue lengths. In Section 6, we numerically validate the accuracy of the approximations and present additional results which give insight in the delay of the network. Section 7 concludes the paper. Proofs of our results are given in the Appendix.

\section{MODEL}

We consider a tandem model consisting of $N$ first-in-first-out (FIFO) systems with unlimited queue, $Q_{i}, i=1, \ldots, N$, in which customers arrive to $Q_{1}$ and subsequently require service at $Q_{2}, Q_{3}, \ldots$, and $Q_{N-1}$ before reaching their destination at $Q_{N}$. The special feature of 


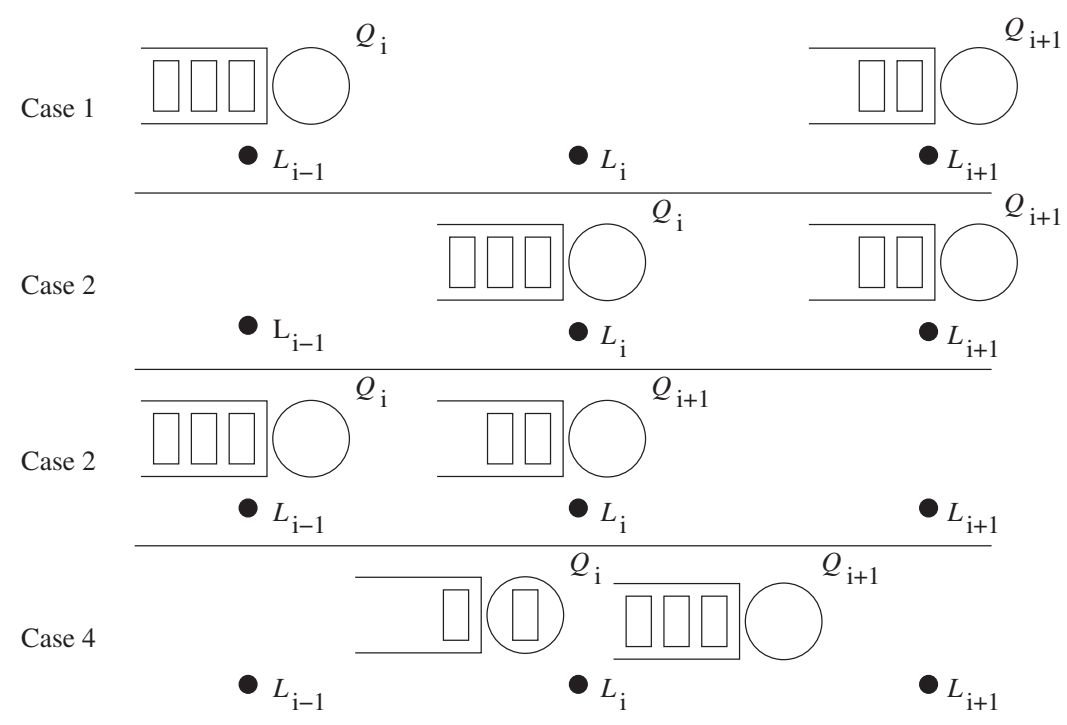

Figure 1. Possible locations of $Q_{i}$ and $Q_{i+1}$.

the model is that $Q_{i}, i=2, \ldots, N-1$, alternates between positions $L_{i-1}$ and $L_{i}$ such that $Q_{i-1}$ 's server is available for service (i.e., customers at $Q_{i-1}$ are served) only when both $Q_{i-1}$ and $Q_{i}$ are at $L_{i-1}$ and $Q_{i}$ 's server is available for service when both $Q_{i}$ and $Q_{i+1}$ are at $L_{i}$. The servers of $Q_{i-1}$ and $Q_{i}$ are two different servers that cannot be serving at the same time. $Q_{1}$ and $Q_{N}$ are fixed and they remain at location $L_{1}$ and $L_{N-1}$ respectively. $Q_{N}$ is a sink and will not be included in our analysis.

Customers arrive to $Q_{1}$ according to a Poisson process with arrival rate $\lambda$. The service requirement $B_{i}$ at $Q_{i}$ has general distribution $B_{i}(t)$ with mean $b_{i}$. We assume that the service requirements are independent and identically distributed (i.i.d.) random variables (rvs).

The queues $Q_{i}, i=2, \ldots, N-1$, move autonomously. $Q_{i}$ remains at location $L_{i-1}$ (resp. $\left.L_{i}\right)$ a random duration $X_{i, n}^{i-1}\left(\operatorname{resp} . X_{i, n}^{i}\right)$ before it migrates to $L_{i}\left(\right.$ resp. $\left.L_{i-1}\right)$ during its $n$th visit, see Figure 1 . The location of $Q_{i}$ is driven by an underlying continuous-time, discretestate, process $\left\{L_{i}(t): t \geq 0\right\}$ with state-space $\{0,1\}$ with $L_{i}(t)=1\left(L_{i}(t)=0\right)$ when $Q_{i}$ is at $L_{i-1}\left(\right.$ resp. $\left.L_{i}\right)$ at time $t$. Without loss of generality, let $L_{i}(0)=1$. We assume $\left\{X_{i, n}^{i-1}, X_{i, n}^{i}\right\}$ i.i.d. and mutually independent, and also independent of the inter-arrival times and service requirements. We further assume that $X_{i, n}^{i-1}\left(X_{i, n}^{i}\right)$ is an i.i.d. sequence of exponentially distributed rvs with rate $\alpha_{i}^{1}\left(\alpha_{i}^{0}\right)$.

We will refer to the time period during which the server is available for service at $Q_{i}$ as $Q_{i}$ service period. Due to the tandem structure, the $Q_{i}$ service period represents the $Q_{i+1}$ arrival period, that is, the period of time during which $Q_{i+1}$ receives customers that completed their service at $Q_{i}$. The $Q_{i}$ service period occurs when the process $\left(L_{i}(t), L_{i+1}(t)\right)$ is in state $(0,1)$, see Case 4 in Figure 1 . The duration of the $n$th $Q_{i}$ service period, denoted by $Y_{i, n}$, is the minimum of the exponentially distributed $\operatorname{rvs} X_{i, m}^{i}$ and $X_{i+1, l}^{i}$, for some $m$ and $l$. That is, $Y_{i, n}$ is an i.i.d. sequence of exponentially distributed rvs with rate $\xi_{i}:=\alpha_{i}^{0}+\alpha_{i+1}^{1}$. Let $Y_{i}$ denote the generic rv of $Y_{i, n}$. During a $Q_{i}$ service period, the server alternates between service and idle states depending on whether or not customers are present at $Q_{i}$. When the server is serving a customer at the end of a server visit to $Q_{i}$, service will be preempted. 
At the beginning of the next visit to $Q_{i}$, the service time will be re-sampled according to $B_{i}(\cdot)$. This discipline is commonly referred to as preemptive-repeat-random.

In the following, given a continuous rv $X, X(t)$ will denote its distribution function, $\tilde{X}(s)$ its Laplace-Stieltjes transform (LST) and $x$ its expectation. Similarly, given a discrete rv $Y, Y(n)$ will denote its distribution function, $\hat{Y}(z)$ its probability generating function (p.g.f.) and $y$ its expectation.

The preemptive-repeat-random discipline induces that the amount of work generated by a customer to $Q_{i}$, referred to as generalized work, can be written as

$$
B_{i}^{g}=B_{i}^{*}+\sum_{l=1}^{L} Y_{i, l}^{*},
$$

where $B_{i}^{*}$ is the conditional $Q_{i}$ service time given that it is smaller than $Q_{i}$ service period, $L$ is the total number of interruptions during the customer's service, and $Y_{i, l}^{*}$ is the conditional $Q_{i}$ service period given that it is smaller than $Q_{i}$ service time. Since a $Q_{i}$ service period is exponentially distributed, it is easily seen that the distribution of $L$ is geometric with mean $\left(\mathbb{P}\left[B_{i}>Y_{i}\right]\right)^{-1}=\left(\tilde{B}_{i}\left(\xi_{i}\right)\right)^{-1}$. Conditioning on $L$, the LST of generalized work is

$$
\tilde{B}_{i}^{g}(s)=\frac{\left(s+\xi_{i}\right) \tilde{B}_{i}\left(s+\xi_{i}\right)}{s+\xi_{i} \tilde{B}_{i}\left(s+\xi_{i}\right)}, \quad \operatorname{Re}(s) \geq 0,
$$

where $\operatorname{Re}(s)$ denotes the real part of $s$. In particular, its expectation reads

$$
\mathbb{E}\left[B_{i}^{g}\right]=\frac{1-\tilde{B}_{i}\left(\xi_{i}\right)}{\xi_{i} \tilde{B}_{i}\left(\xi_{i}\right)} .
$$

Let $N_{i}(t)$ denote the number of customers in $Q_{i}, i=1, \ldots, N$, at time $t$. Assume that $N_{i}(0)=0, i=1, \ldots, N$. Let $D_{i}$ denote the sojourn time of an arbitrary customer in $Q_{i}$, $i=1, \ldots, N-1$. In the following, we will study $\tilde{D}_{i}(s)$, the LST of the sojourn time in $Q_{i}$, $i=1, \ldots, N-1$.

\section{STABILITY}

Stability is considered on a per-queue basis as service capacity cannot be exchanged between the queues. The system is stable if and only if all the queues in the system are stable.

For an individual queue to be stable, we must have that the average work per unit time brought by a customer to the queue, $\lambda \mathbb{E}\left[B_{i}^{g}\right]$, is strictly smaller than the average fraction of time the queue server is available for service. In the following, we will derive the average fraction of time the $Q_{i}$ server is available for service, which corresponds to the probability that the $Q_{i}$ server is available. At time $t$, the $Q_{i}$ server is available when both $Q_{i}$ and $Q_{i+1}$ are at location $L_{i}$, that is, when $\left(L_{i}(t), L_{i+1}(t)\right)=(0,1)$. By a renewal reward argument, we have that

$$
\mathbb{P}\left(L_{i}(t)=k\right)=\frac{\alpha_{i}^{1-k}}{\alpha_{i}^{1}+\alpha_{i}^{0}}, \quad k=0,1,
$$

and since the mobility processes of $Q_{i}$ and $Q_{i+1}$ are independent, we obtain that

$$
\begin{aligned}
\mathbb{P}\left(\left(L_{i}(t), L_{i+1}(t)\right)=(0,1)\right) & =\mathbb{P}\left(L_{i}(t)=0\right) \mathbb{P}\left(L_{i+1}(t)=1\right) \\
& =\frac{\alpha_{i}^{1} \alpha_{i+1}^{0}}{\left(\alpha_{i}^{1}+\alpha_{i}^{0}\right)\left(\alpha_{i+1}^{1}+\alpha_{i+1}^{0}\right)} .
\end{aligned}
$$


Note that $Q_{1}$, the source node, remains always at location $L_{1}$, that is, $L_{1}(t)=0, t \geq 0$. This can be included in (5) by letting $\alpha_{1}^{1} \rightarrow \infty$ and $\alpha_{1}^{0}=0$. Moreover, since $Q_{N}$ remains at location $L_{N}$, we let $\alpha_{N}^{1}=0$ and $\alpha_{N}^{0} \rightarrow \infty$, so that (5) is valid for $i=1, \ldots, N$.

\subsection{Stability Condition}

$Q_{i}$ is stable iff

$$
\begin{aligned}
\rho_{i} & :=\frac{\lambda \mathbb{E}\left[B_{i}^{g}\right]}{\mathbb{P}\left(\left(L_{i}(t), L_{i+1}(t)\right)=(0,1)\right)} \\
& =\lambda \frac{1-\tilde{B}_{i}\left(\xi_{i}\right)}{\xi_{i} \tilde{B}_{i}\left(\xi_{i}\right)}\left(\frac{\left(\alpha_{i}^{1}+\alpha_{i}^{0}\right)\left(\alpha_{i+1}^{1}+\alpha_{i+1}^{0}\right)}{\alpha_{i}^{1} \alpha_{i+1}^{0}}\right)<1,
\end{aligned}
$$

where $\rho_{i}$ is referred to as the generalized load at $Q_{i}$.

Note that under stability the arrival rates to $Q_{i+1}$ and $Q_{i}$ are equal.

\section{EXACT ANALYSIS OF QUEUE ONE}

The server visit process is autonomous and the service is according to the preemptive-repeatrandom discipline. It is then easily seen that $Q_{1}$ in isolation is an $M / G / 1$ queue with on-off server with arrival rate $\lambda$, mean service time $b_{1}$, exponential on-period $X_{2}^{1}$ with rate $\alpha_{2}^{1}$, and off-period $R^{\text {off }}$ equal to the $Q_{2}$ sojourn time at $L_{2}$, that is, $R^{\text {off }}=X_{2}^{2}$. By a renewal reward argument, $P_{\text {on }}$, the probability that the server is on, satisfies $P_{\text {on }}=\mathbb{P}\left(L_{2}(t)=1\right)$ which is given in (4), and $P_{\text {off }}:=1-P_{\text {on }}$.

The $M / G / 1$ queue with on-off server has extensively been studied in the literature (see, e.g., $[9,15])$. Let us state here only the results that are relevant for our analysis. The LST of the sojourn time of a customer is denoted by $\tilde{D}_{1}(s)$ and follows from a decomposition argument [15]

$$
\tilde{D}_{1}(s)=\tilde{W}_{1}(s) \tilde{B}_{1}^{\text {eff }}(s),
$$

where $\tilde{W}_{1}(s)$ and $\tilde{B}_{1}^{\text {eff }}(s)$ denote the LST of the waiting time of a customer (until it is taken into service for the first time) and the effective service time (including possible service interruptions), respectively. Note that $B_{1}^{\text {eff }}$ includes the service interruption time and is therefore not equal to $B_{1}^{g}$. The LSTs $\tilde{W}_{1}(s)$ and $\tilde{B}_{1}^{\text {eff }}(s)$ are given by [1]

$$
\begin{aligned}
\tilde{W}_{1}(s) & =\tilde{W}_{M / G / 1}(s)\left(P_{o n}+P_{o f f} \tilde{R}_{e}^{\text {off }}(s)\right), \\
\tilde{B}_{1}^{\mathrm{eff}}(s) & =\frac{\left(\alpha_{2}^{1}+s\right)\left(\alpha_{2}^{0}+s\right) \cdot \tilde{B}_{1}\left(\alpha_{2}^{1}+s\right)}{\left(\alpha_{2}^{1}+s\right)\left(\alpha_{2}^{0}+s\right)-\alpha_{2}^{1} \alpha_{2}^{0}\left(1-\tilde{B}_{1}\left(\alpha_{2}^{1}+s\right)\right)},
\end{aligned}
$$

where $\operatorname{Re}(s) \geq 0, \quad \tilde{R}_{e}^{\text {off }}(s)$ denotes the LST of the residual time of an off-period, and $\tilde{W}_{M / G / 1}(s)$ is the LST of the waiting time in the corresponding $M / G / 1$ queue with service time with LST $\tilde{B}_{1}^{\text {eff }}(s)$.

It follows that $\hat{N}_{1}(z)$, the p.g.f. of the $Q_{1}$ queue length, can be expressed as function of $\tilde{D}_{1}(s)$, using the so-called functional form of Little's law, as follows (see, e.g., [19])

$$
\hat{N}_{1}(z)=\tilde{D}_{1}(\lambda(1-z)), \quad|z| \leq 1 .
$$

Let us denote by $\hat{N}_{1}^{v}(z)$ the p.g.f. of $Q_{1}$ queue length at the start time of its service period. It can then be shown using Eq. (10), the PASTA property and conditioning on the position 
of the server, that

$$
\hat{N}_{1}^{v}(z)=\tilde{W}_{M / G / 1}(\lambda(1-z)) \cdot \tilde{B}_{1}^{\mathrm{eff}}(\lambda(1-z)) \cdot \tilde{R}_{e}^{\mathrm{off}}(\lambda(1-z))
$$

Moreover, let $K_{2, n}$ denote the total number of arrivals to $Q_{2}$ during its $n$th arrival period. Since in our tandem model two successive queues cannot be on service at the same time, the results derived for the p.g.f. of the joint queue length in a time-limited polling model in $[2,6]$ can be used to find that

$$
\hat{K}_{2, n}(z)=\frac{1}{1-z \tilde{B}_{1}\left(\alpha_{2}^{1}\right)}\left[1-\tilde{B}_{1}\left(\alpha_{2}^{1}\right)+\frac{\alpha_{2}^{1} \tilde{B}_{1}\left(\alpha_{2}^{1}\right)(1-z)}{\alpha_{2}^{1}+\lambda\left(1-\mu\left(\alpha_{2}^{1}, z\right)\right)} \hat{N}_{1}^{v}\left(\mu\left(\alpha_{2}^{1}, z\right)\right)\right],
$$

where $\mu\left(\alpha_{2}^{1}, z\right)$ is the smallest root of $x=z \tilde{B}_{1}\left(\alpha_{2}^{1}+\lambda(1-x)\right)$ with $\left|\mu\left(\alpha_{2}^{1}, z\right)\right|<1 . \hat{K}_{2, n}(z)$ will be required later in the approximative analysis for $Q_{2}$. We note that the related asymptotic results of the variance of departures in critically loaded single-server queues is analyzed in [3]. In the following, we will study each mobile queue in isolation.

\section{SOJOURN TIME APPROXIMATION VIA QUEUE LENGTH}

In this section, we present an approximation for the LST of the sojourn time of a customer in $Q_{i}$, denoted by $\tilde{D}_{i}(s), i=2, \ldots, N-1$, via queue lengths. We refer to this approximation as the queue-length approximation. We consider the queue-length process of $Q_{i}$ when $\left(L_{i}(t), L_{i+1}(t)\right)=(0,1)$, that is, during a $Q_{i}$ service period. It turns out that this queue-length process corresponds to the waiting time in a $\mathrm{Geo} / \mathrm{G} / 1$ discrete-time queue with geometric inter-arrival time distribution and general service requirement distribution. The delay $D_{i}$ follows from adding the total time a customer spends in service to the latter waiting time.

\subsection{Queue Length of $Q_{i}$}

Consider the queue-length process of $Q_{i}$ only during its service periods. This is done by removing the time intervals where the $Q_{i}$ server is not present, that is, during $Q_{i}$ off-periods. This new process can be seen as the queue length in a batch arrival queue with inter-arrival times distributed as $Q_{i}$ service period. Let $y_{n}, n=0,1, \ldots$, denote the ending times of $Q_{i}$ service periods. Let $N_{i, n}^{e}$ denote the queue length of $Q_{i}$ at epoch $y_{n}$. Assume that the queue length is left-continuous, that is, arriving batches are not counted as being in the system until (just) after they arrive.

Let $M_{n}$ denote the total number of $Q_{i}$ arrival periods that occur between the $n$th and $(n+1)$-st $Q_{i}$ service period. Note that due to the tandem structure in our model it is clear that the $Q_{i}$ arrival period represents the $Q_{i-1}$ service period. Let $K_{i, n}^{m}$ denote the total number of arrivals to $Q_{i}$ during the $m$ th $Q_{i}$ arrival period for $m=1, \ldots, M_{n}$. Thus, between the end of the $n$th and $(n+1)$-st service period $\sum_{m=1}^{M_{n}} K_{i, n}^{m}$ customers arrive to $Q_{i}$. So that, in our interpretation of the batch arrival queue with off-service periods removed, at time $y_{n}$ a batch of size $\sum_{m=1}^{M_{n}} K_{i, n}^{m}$ arrives to the queue. Note that it is possible that $M_{n}=0$, in this case the batch size is simply equal to zero. Let $E_{i, n+1}$ denote the number of customers that complete their service in $Q_{i}$ during the $(n+1)$-st service period in the case where at the beginning of this period the $Q_{i}$ queue length is infinite. A sample path of the evolution of $N_{i}(t)$ as a function of $t$ is depicted in Figure 2. It is then easily seen that 


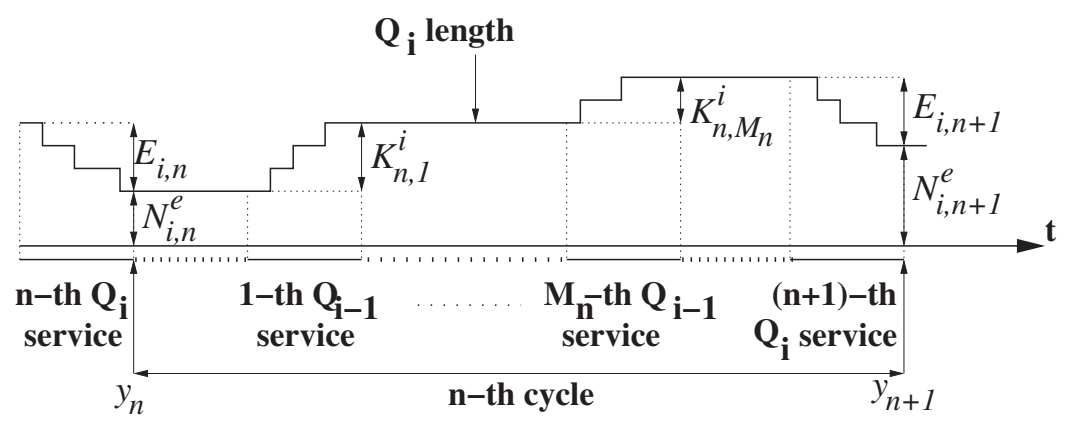

Figure 2. Sample path of the $Q_{i}$ queue length.

during the $n$th cycle, $\left[y_{n}, y_{n+1}\right), N_{i, n+1}^{e}$ can be written as function of $N_{i, n}^{e}$ as follows:

$$
N_{i, n+1}^{e}=\left(N_{i, n}^{e}+\sum_{m=1}^{M_{n}} K_{i, n}^{m}-E_{i, n+1}\right)^{+}, \quad n \geq 0 .
$$

where $(\cdot)^{+}:=\max (0, \cdot)$. Recall that $Y_{i, n+1}$ denotes the duration of the $(n+1)$-st $Q_{i}$ service period that is an exponentially distributed $\mathrm{rv}$ with rate $\xi_{i}=\alpha_{i}^{0}+\alpha_{i+1}^{1}$. Recall that the customers service requirements are i.i.d. rvs with general distribution. It follows that $E_{i, n}$, $n=0,1, \ldots$, are i.i.d. rvs, which are geometrically distributed with parameter $\nu_{i}:=\mathbb{P}\left(B_{i}<\right.$ $\left.Y_{i, n+1}\right)=\tilde{B}_{i}\left(\xi_{i}\right)$. Thus, the probability of the event $\left\{E_{i, n+1}=l\right\}$ reads

$$
\mathbb{P}\left(E_{i, n+1}=l\right)=\left(1-\nu_{i}\right) \nu_{i}^{l}, \quad l=0,1, \ldots .
$$

Note that $E_{i, n+1}$ is independent of $K_{i, n}^{m}$ and $M_{n}, K_{i, n}^{m}$ depends on the index $m$, however, it is independent of the rv $M_{n}$, and that $K_{i, n}^{m}$ depends on the queue-length process of $Q_{i-1}$ during the time interval between the $n$th and $(n+1)$-st $Q_{i}$ service periods. Moreover, the queue length of $Q_{i-1}$ does not form a Markov chain. For this reason, the rvs $K_{i, n}^{m}, n=0,1, \ldots$, $m=1, \ldots, M_{n}$, are not independent rvs. In addition, $E_{i, n}$ and $K_{i, n}^{m}$ are not independent. For the sake of model tractability, we make the following approximating assumption:

Assumption A: $K_{i, n}^{m}, n=0,1, \ldots, m=1, \ldots, M_{n}$ are i.i.d. and also independent of $\left\{E_{i, l}\right.$ : $l=0,1, \ldots, n\}$.

By Assumption A, Eq. (13) represents the waiting time of an arrival in a discretetime single-server queue with inter-arrival times $E_{i, n+1}$ and service requirements $F_{i, n}:=$ $\sum_{m=1}^{M_{n}} K_{i, n}^{m}$. The main advantage in this model is that the distribution of $E_{i, n+1}$ is geometric. It is known that $\hat{N}_{i}^{e}(z)$, the steady-state p.g.f. of $N_{i, n}^{e}$, is given by (see [16, Corollary 4.3] with $U$ and $B$ equal in distribution and $\gamma=p=\nu_{i}$ )

$$
\hat{N}_{i}^{e}(z)=\frac{\left(1-\nu_{i}-\nu_{i} \mathbb{E}\left[F_{i}\right]\right)(z-1)}{z-1+\nu_{i}\left(1-z \hat{F}_{i}(z)\right)}, \quad|z| \leq 1,
$$

where $\hat{F}_{i}(z)$ is the steady-state p.g.f. of $F_{i, n}$. Since $K_{i, n}^{m}$ is independent of $M_{n}$, the p.g.f. $\hat{F}_{i}(z)$ can be written as follows:

$$
\hat{F}_{i}(z)=\mathbb{E}\left[\mathbb{E}\left[z^{K_{i, n}}\right]^{M_{n}}\right]=\hat{M}_{n}\left(\hat{K}_{i, n}(z)\right) .
$$

We emphasize that $\hat{K}_{i, n}(z)$ follows from the analysis of $Q_{i-1}$. For this reason, to complete the analysis of $Q_{i}$, in Section 5.4 we will derive $\hat{K}_{i+1, n}(z)$. 
To derive the LST of the sojourn time at $Q_{i}$ we need $\hat{N}_{i}^{c}(z)$, the p.g.f. of $Q_{i}$ queue length seen by an arbitrary customer, and $\hat{M}_{n}(z)$, which will be determined in Section 5.2.

LEMMA 1: The p.g.f. of the queue length of $Q_{i}$ seen by an arbitrary arriving customer is given by

$$
\hat{N}_{i}^{c}(z)=\hat{N}_{i}^{e}(z) \frac{z\left(1-\hat{F}_{i}(z)\right)}{(1-z) \mathbb{E}\left[F_{i}\right]}
$$

Proof: Let $\hat{N}_{i}^{j}(z)$ denote the p.g.f. of $Q_{i}$ queue length seen by the $j$ th customer within a batch upon arrival including himself. Since the size of the batches is independent of the queue length of $Q_{i}$ present upon arrival, $\hat{N}_{i}^{j}(z)$ reads,

$$
\hat{N}_{i}^{j}(z)=z \hat{N}_{i}^{j-1}(z), \quad j=1,2, \ldots
$$

with $\hat{N}_{i}^{0}(z)=\hat{N}_{i}^{e}(z)$. The probability, $\mathbb{P}(J=j)$, that a customer is the $j$ th customer within the batch is equal to the fraction of customers who are $j$ th arrival in their own batch, which gives

$$
\mathbb{P}(J=j)=\frac{\mathbb{P}\left(F_{i} \geq j\right)}{\mathbb{E}\left[F_{i}\right]} .
$$

Removing the condition on the customer position in a batch in (18) and using (19) gives the desired result.

As can be seen $\hat{N}_{i}^{c}(z)$ is function of $\hat{F}_{i}(z)$ and eventually of $\hat{M}_{n}(z)$. Now, we derive $\hat{M}_{n}(z)$.

\subsection{P.g.f. of $M_{n}$}

The rv $M_{n}$ only depends on the mobility process of $Q_{i-1}, Q_{i}$, and $Q_{i+1}$ and can be fully represented as the number of visits to a state in a Markov chain.

The p.g.f. of $M_{n}$ can be written as follows:

$$
\hat{M}_{n}(z)=\mathbb{P}\left(M_{n}=0\right)+\left(1-\mathbb{P}\left(M_{n}=0\right)\right) \hat{M}_{n}^{+}(z),
$$

where $M_{n}^{+}$is $M_{n}$ given that it is strictly positive. In the following lemmas, we will first derive $\hat{M}_{n}^{+}(z)$ and next $\mathbb{P}\left(M_{n}=0\right)$.

Lemma 2: The p.g.f. of $M_{n}^{+}$is

$$
\hat{M}_{n}^{+}(z)=-b z(\mathbf{A}+z \mathbf{B})^{-1} u, \quad|z| \leq 1
$$


where

$$
\begin{aligned}
\mathbf{A} & =\left(\begin{array}{cccccc}
A_{11} & 0 & 0 & \alpha_{i-1}^{0} & 0 & 0 \\
\alpha_{i}^{1} & A_{22} & \alpha_{i+1}^{0} & 0 & \alpha_{i-1}^{0} & 0 \\
0 & \alpha_{i+1}^{1} & A_{33} & 0 & 0 & \alpha_{i-1}^{0} \\
\alpha_{i-1}^{1} & 0 & 0 & A_{44} & \alpha_{i}^{0} & 0 \\
0 & 0 & 0 & \alpha_{i}^{1} & A_{55} & \alpha_{i+1}^{0} \\
0 & 0 & 0 & 0 & \alpha_{i+1}^{1} & A_{66}
\end{array}\right), \\
\mathbf{B} & =\left(\begin{array}{cccccc}
0 & \alpha_{i}^{0} & 0 & 0 & 0 & 0 \\
0 & 0 & 0 & 0 & 0 & 0 \\
0 & 0 & 0 & 0 & 0 & 0 \\
0 & 0 & 0 & 0 & 0 & 0 \\
0 & \alpha_{i-1}^{1} & 0 & 0 & 0 & 0 \\
0 & 0 & \alpha_{i-1}^{1} & 0 & 0 & 0
\end{array}\right), \quad u=\left(\begin{array}{c}
\alpha_{i+1}^{0} \\
0 \\
\alpha_{i}^{1} \\
\alpha_{i+1}^{0} \\
0 \\
\alpha_{i}^{1}
\end{array}\right),
\end{aligned}
$$

and where the diagonal entries of $\mathbf{A}$ are such that $(\mathbf{A}+\mathbf{B}) e+u=\mathbf{0}$. The vector $b$ is the row vector of order six and of non-zero entries

$$
\begin{aligned}
b(2)= & h(1), \quad b(3)=1-h(1), \\
h & =-\frac{\left(\alpha_{i-1}^{1} \alpha_{i+1}^{1}, 0, \alpha_{i-1}^{0} \alpha_{i+1}^{1}, 0,0, \alpha_{i-1}^{0} \alpha_{i}^{0}\right)}{\left(\alpha_{i-1}^{0}+\alpha_{i-1}^{1}\right)\left(\alpha_{i}^{0}+\alpha_{i+1}^{1}\right)} \cdot \mathbf{H}^{-1} \cdot \mathbf{V}, \\
\mathbf{H} & =\left(\begin{array}{cccccc}
H_{11} & \alpha_{i+1}^{0} & \alpha_{i-1}^{0} & 0 & 0 & 0 \\
\alpha_{i+1}^{1} & H_{22} & 0 & \alpha_{i-1}^{0} & 0 & 0 \\
\alpha_{i-1}^{1} & 0 & H_{33} & \alpha_{i+1}^{0} & \alpha_{i}^{0} & 0 \\
0 & \alpha_{i-1}^{1} & \alpha_{i+1}^{1} & H_{44} & 0 & \alpha_{i}^{0} \\
0 & 0 & \alpha_{i}^{1} & 0 & H_{55} & \alpha_{i+1}^{0} \\
0 & 0 & 0 & \alpha_{i}^{1} & \alpha_{i+1}^{1} & H_{66}
\end{array}\right), \mathbf{V}=\left(\begin{array}{cc}
\alpha_{i}^{0} & 0 \\
0 & \alpha_{i}^{0} \\
0 & 0 \\
0 & 0 \\
\alpha_{i-1}^{1} & 0 \\
0 & \alpha_{i-1}^{1}
\end{array}\right) .
\end{aligned}
$$

The diagonal entries of $\mathbf{H}$ are such that $\mathbf{H} e+\mathbf{V} \times(1,1)^{T}=\mathbf{0}$, where $e$ is the column vector of order six and with all entries equal to 1 , and $x^{T}$ is the transpose of vector $x$.

Proof: See Appendix A.1.

Lemma 3: The probability that no $Q_{i}$ arrival period occurs during the $n$th cycle reads

$$
\mathbb{P}\left(M_{n}=0\right)=-g \cdot \mathbf{F}^{-1} \cdot w
$$

where

$$
\begin{aligned}
g & =\frac{1}{\left(\alpha_{i-1}^{0}+\alpha_{i-1}^{1}\right)\left(\alpha_{i}^{0}+\alpha_{i+1}^{1}\right)}\left(\alpha_{i-1}^{1} \alpha_{i+1}^{1}, \alpha_{i-1}^{0} \alpha_{i+1}^{1}, 0, \alpha_{i-1}^{0} \alpha_{i}^{0}\right), \\
\mathbf{F} & =\left(\begin{array}{cccc}
F_{11} & \alpha_{i-1}^{0} & 0 & 0 \\
\alpha_{i-1}^{1} & F_{22} & \alpha_{i}^{0} & 0 \\
0 & \alpha_{i}^{1} & F_{33} & \alpha_{i+1}^{0} \\
0 & 0 & \alpha_{i+1}^{1} & F_{44}
\end{array}\right), \quad w=\left(\begin{array}{c}
\alpha_{i+1}^{0} \\
\alpha_{i+1}^{0} \\
0 \\
\alpha_{i}^{1}
\end{array}\right) .
\end{aligned}
$$


The diagonal entries of $\mathbf{F}$ are such that

$$
\mathbf{F} e+w+\left(\alpha_{i}^{0}, 0, \alpha_{i-1}^{1}, \alpha_{i-1}^{1}\right)^{T}=\mathbf{0} .
$$

Proof: See Appendix A.2.

\subsection{Sojourn Time in $Q_{i}$}

Recall that $D_{i}$, the sojourn time in $Q_{i}$, consists of two parts: the time required to serve $N_{i}^{c}$ customers, and the time a customer is in $Q_{i}$ but $Q_{i}$ is not served. Let $B_{i}^{\text {eff }}$ denote the effective service time at $Q_{i}, i=2, \ldots, N-1$, that starts when a customer receives the service for the first time and ends when the customer departs from $Q_{i}$. Clearly, $B_{i}^{\text {eff }}$ includes the time when the $Q_{i}$ service is interrupted. Let $L$ denote the total number of interruptions during the service of a customer. It is easily seen that $B_{i}^{\text {eff }}$ can be written as

$$
B_{i}^{\mathrm{eff}}=B_{i}^{*}+\sum_{l=1}^{L}\left(Y_{i, l}^{*}+\Xi_{i, l}\right)
$$

where $B_{i}^{*}$ is the conditional $B_{i}$ given that it is smaller than $Y_{i}$, the exponential rv with rate $\xi_{i}=\alpha_{i}^{0}+\alpha_{i+1}^{1}, Y_{i, l}^{*}$ is the conditional $Y_{i}$ given that it is smaller than $B_{i}$, and $\Xi_{i, l}$ is the duration of the service interruption in $Q_{i}$. Let $\tilde{\Xi}_{i}(s)$ denote the steady-state LST of $\Xi_{i, l}$. Since we are considering the preemptive-repeat discipline, the distribution of $L$ is geometric with parameter $\mathbb{P}\left[B_{i}>Y_{i}\right]=1-\tilde{B}_{i}\left(\xi_{i}\right)$. Conditioning on $L$, we find the LST of $B_{i}^{\text {eff }}$ that reads

$$
\tilde{B}_{i}^{\text {eff }}(s)=\frac{\left(\xi_{i}+s\right) \cdot \tilde{B}_{i}\left(\xi_{i}+s\right)}{\left(\xi_{i}+s\right)-\xi_{i}\left(1-\tilde{B}_{i}\left(\xi_{i}+s\right)\right) \tilde{\Xi}_{i}(s)}, \quad i=2, \ldots, N-1, \quad \operatorname{Re}(s) \geq 0 .
$$

An arriving customer to $Q_{i}$ joins the queue when $Q_{i}$ is not served. Therefore, the customer has to wait for the server to return to $Q_{i}$ in order that her service starts. This occurs when $Q_{i}$ and $Q_{i+1}$ are both at location $L_{i}$. Let $\Xi_{i}^{*}$ denote the first time after $t$ that the server returns to $Q_{i}$ given that an arrival joins $Q_{i}$ at $t$. In the following lemmas, we give the LST of $\Xi_{i}$ and $\Xi_{i}^{*}$.

LEMMA 4: The LST of $\Xi_{i}$ is

$$
\tilde{\Xi}_{i}(s)=y(s \mathbf{I}-\mathbf{A}-\mathbf{B})^{-1} u,, \quad \operatorname{Re}(s) \geq 0,
$$

where

$$
y=\frac{1}{\left(\alpha_{i-1}^{0}+\alpha_{i-1}^{1}\right)\left(\alpha_{i}^{0}+\alpha_{i+1}^{1}\right)}\left(\alpha_{i-1}^{1} \alpha_{i+1}^{0}, 0, \alpha_{i-1}^{1} \alpha_{i}^{0}, \alpha_{i-1}^{0} \alpha_{i+1}^{1}, 0, \alpha_{i-1}^{0} \alpha_{i}^{0}\right),
$$

A, B, and $u$ are given in Lemma 2.

Proof: See Appendix A.3.

LEMma 5: The LST of $\Xi_{i}^{*}$ is

$$
\tilde{\Xi}_{i}^{*}(s)=y^{*}(s \boldsymbol{I}-\boldsymbol{A}-\boldsymbol{B})^{-1} u, \quad \operatorname{Re}(s) \geq 0,
$$


where

$$
y^{*}=\frac{1}{\alpha_{i+1}^{0}+\alpha_{i+1}^{1}}\left(0, \alpha_{i+1}^{1}, \alpha_{i+1}^{0}, 0,0,0\right) .
$$

Proof: See Appendix A.4.

We are now ready to formulate our main result for the sojourn time in $Q_{i}$, the queuelength approximation.

TheOrem 1 Sojourn time via queue length: Under Assumption A, the sojourn time in $Q_{i}$ is

$$
D_{i}=\Xi_{i}^{*}+\sum_{i=1}^{N_{i}^{c}} B_{i}^{\mathrm{eff}}
$$

The $L S T$ of $D_{i}$ reads

$$
\tilde{D}_{i}(s)=\tilde{\Xi}_{i}^{*}(s) \hat{N}_{i}^{c}\left(\tilde{B}_{i}^{\mathrm{eff}}(s)\right), \quad \operatorname{Re}(s) \geq 0 .
$$

Proof: Equation (29) is due to the fact that the queue length of $Q_{i}$ seen by an arriving customer is $N_{i}^{c}$ (including himself) and the customer in service has to wait for $\Xi_{i}^{*}$ before that the service restarts in $Q_{i}$.

Since $N_{i}^{c}$ depends on the history of the Markov chain $\left(L_{i-1}(t), L_{i}(t), L_{i+1}(t)\right)$ and $B_{i}^{\text {eff }}$ depends on the future of $\left(L_{i-1}(t), L_{i}(t), L_{i+1}(t)\right)$ the rvs $N_{i}^{c}$ and $B_{i}^{\text {eff }}$ are independent. Moreover, $B_{i}^{\text {eff }}$ is independent of $\Xi_{i}^{*}$, e.g., see $(23)$, and $N_{i}^{c}$ is independent $\Xi_{i}^{*}$. All these independencies together readily give $(30)$.

Remark 1: For the exponential service times, we note that in [1] we proposed a different approximation of the sojourn time at $Q_{i}$ via the workload analysis in the queue. We emphasize that the queue-length approximation proposed in this paper is much easier to derive and to extend to the general service times distribution.

\subsection{P.g.f. of $K_{i+1, n}$}

In our tandem model, we note that the arrivals to a queue are the departures of the upstream queue. Therefore, to derive the queue length of $Q_{i}$, it is required to first analyze $Q_{i-1}$, and so on. For this reason, we emphasize that in our iterative scheme the p.g.f. $\hat{K}_{i, n}(z)$ should be computed in the analysis of $Q_{i-1}$. Therefore, to close the iteration loop of $Q_{i}$, we will derive in the following $\hat{K}_{i+1, n}(z)$. The rv $K_{i+1, n}$ represents the total number of arrivals to $Q_{i+1}$ during a $Q_{i}$ service period. Let $N_{i}^{v}$ denote the queue length of $Q_{i}$ just after the beginning of a $Q_{i}$ service period. Therefore, $N_{i}^{v}$ is the sum of $N_{i}^{e}$, the queue length of $Q_{i}$ seen by arriving batch, and $F_{i}$, the batch size. Note that during a $Q_{i}$ server period, there are no arrivals to $Q_{i}$ and the distribution of the duration of that server period is $Y_{i}$, an exponential rv with rate $\xi_{i}=\alpha_{i}^{0}+\alpha_{i+1}^{1}$. Consequently, using (12) with $\lambda \rightarrow 0$ and replacing $\alpha_{2}^{1}$ by $\xi_{i}$ and $\tilde{B}_{1}(s)$ by $\tilde{B}_{i}(s)$ gives

$$
\hat{K}_{i+1, n}(z)=\frac{1}{1-\tilde{B}_{i}\left(\xi_{i}\right) z}\left[\tilde{B}_{i}\left(\xi_{i}\right)(1-z) \hat{N}_{i}^{v}\left(\tilde{B}_{i}\left(\xi_{i}\right) z\right)+1-\tilde{B}_{i}\left(\xi_{i}\right)\right]
$$

where $\hat{N}_{i}^{v}(z):=\hat{N}_{i}^{e}(z) \hat{F}_{i}(z)$, which are given in (15) and (16). 


\section{NUMERICAL RESULTS}

We consider a tandem network of $N$ queues including the source and the destination queue. The mean service time at $Q_{i}$ is equal to $b_{i}=1$ for $i=1, \ldots, N$. Recall that $Q_{i}$ remains at locations $L_{i-1}$ and $L_{i}$ an exponentially distributed period of time with rate $\alpha_{i}^{1}$ and $\alpha_{i}^{0}$. We will consider the case where $\alpha_{i}^{0}=\alpha_{i}^{1}=\alpha_{i}$. The queues $Q_{1}$ and $Q_{N}$ remain always at locations $L_{1}$ and $L_{N}$, respectively. Our objectives are to validate the approximations and to give insights into the sojourn time behavior as a function of the system parameters.

To validate our approximation we will compare its results with those of the simulation. The simulation of the above tandem model scenario was implemented in the $\mathrm{C}++$ programming language. To generate the rvs we used the pseudo-random generator package of $\mathrm{C}++$. We note that a simulation result consists of an average over multiple runs with different seeds. The number of runs considered is high enough in order to guarantee a small $95 \%$ confidence interval.

Let $\mathbb{E}\left[D_{i}^{\mathrm{ql}}\right]$ denote the mean sojourn time in $Q_{i}$ using the queue-length approximation given in (30). Let $\mathbb{E}\left[D_{i}^{\operatorname{sim}}\right]$ denote the mean sojourn time in $Q_{i}$ using simulation for the tandem network. Moreover, let us refer to the relative difference between the mean sojourn time at $Q_{i}$ using the approximation and simulation as follows.

$$
\tau_{i}^{\mathrm{ql}}(\%):=100 \times\left(1-\frac{\mathbb{E}\left[D_{i}^{\mathrm{ql}}\right]}{\mathbb{E}\left[D_{i}^{\mathrm{sim}}\right]}\right) .
$$

In the sequel, we will consider four different service time distributions: the deterministic distribution, the Erlang-2 distribution, the exponential distribution, and the two-phase hyper-exponential distribution. The two-phase hyper-exponential distribution is uniquely determined by its mean value $b$, and by the mean $m_{1}$ and probability $p_{1}$ of the first phase. In the next section, we present some general error analysis by varying all parameters. This is followed by more detailed studies focussing on only one parameter at a time.

\subsection{Global Accuracy Results of the Queue-Length Approximation}

In this section, we study the effects of varying several parameters like the maximal load $\rho$ at the stations, the visit time parameter $\alpha$ and the SCV of the underlying service times on the performance for tandem queues of various length. As performance measure we take the sojourn time in the last mobile queue. In our numerical experiments, this last mobile queue is the one with the largest relative error (see section 6.2). In Figures 3 and 4 , we present $\tau_{N-1}^{\mathrm{ql}}(\%)$, the percentual error of our approximation for this sojourn time. We take the number of nodes in the tandem queue $N \in\{4,7,10\}$, the mean service time at every node $b_{i}=1$ and the squared coefficient of variation (SCV) of the service times $\mathrm{SCV} \in\{0,0.5,1,3.43\}$. Furthermore, we consider the system with $\alpha_{i}=0.025$ (long visits to every node), $\alpha_{i}=0.2$, (short visits), $\alpha_{i}=0.025+\frac{i-1}{N-2}(0.2-0.025)$ (decreasing visit times) or $\alpha_{i}=0.2-\frac{i-1}{N-2}(2-0.025)$ (increasing visit times) for $i=2, \ldots, N-1$. Finally, for every combination of $N, \mathrm{SCV}$ and $\alpha_{i}$ the arrival rate $\lambda$ is varied such that the maximal load at a node $\rho \in\{0.1,0.25,0.4,0.55,0.7\}$ (see Eq. (6)). So, in total, we analyzed 240 different scenarios. The results indicate that the approximation is not very sensitive to the SCV of the service times. On the other hand, the approximation is very sensitive to the visit time parameter $\alpha$; especially when the visit times are very long compared to the service time the approximation reaches its limit. Overall, the approximation works well when $\rho=0.25$ but also for $\rho=0.4$ and the $\alpha^{\prime}$ s not to small. For larger values of $\rho$ or $\alpha$, the accuracy of the approximation drops significantly. Here, we also remark that taking another performance 


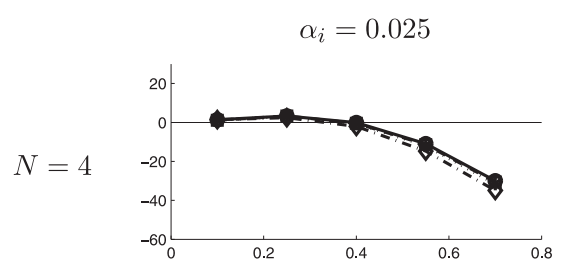

$$
\alpha_{i}=0.2-\frac{i-1}{N-2}(2-0.025)
$$
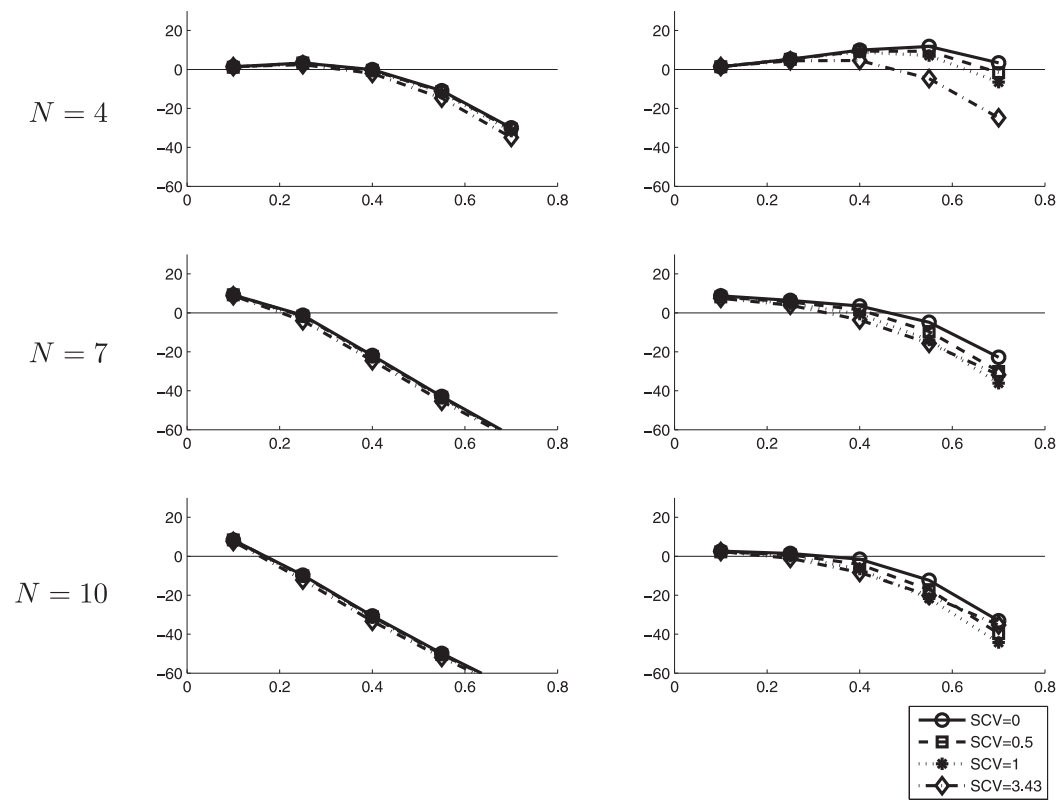

Figure 3. Relative errors (\%) in the sojourn time of the last mobile node (on the vertical axis) as function of the maximal load (on the horizontal axis) for $\alpha_{i}=0.025$ and $\alpha_{i}=0.2-$ $\frac{i-1}{N-2}(2-0.0025)$.

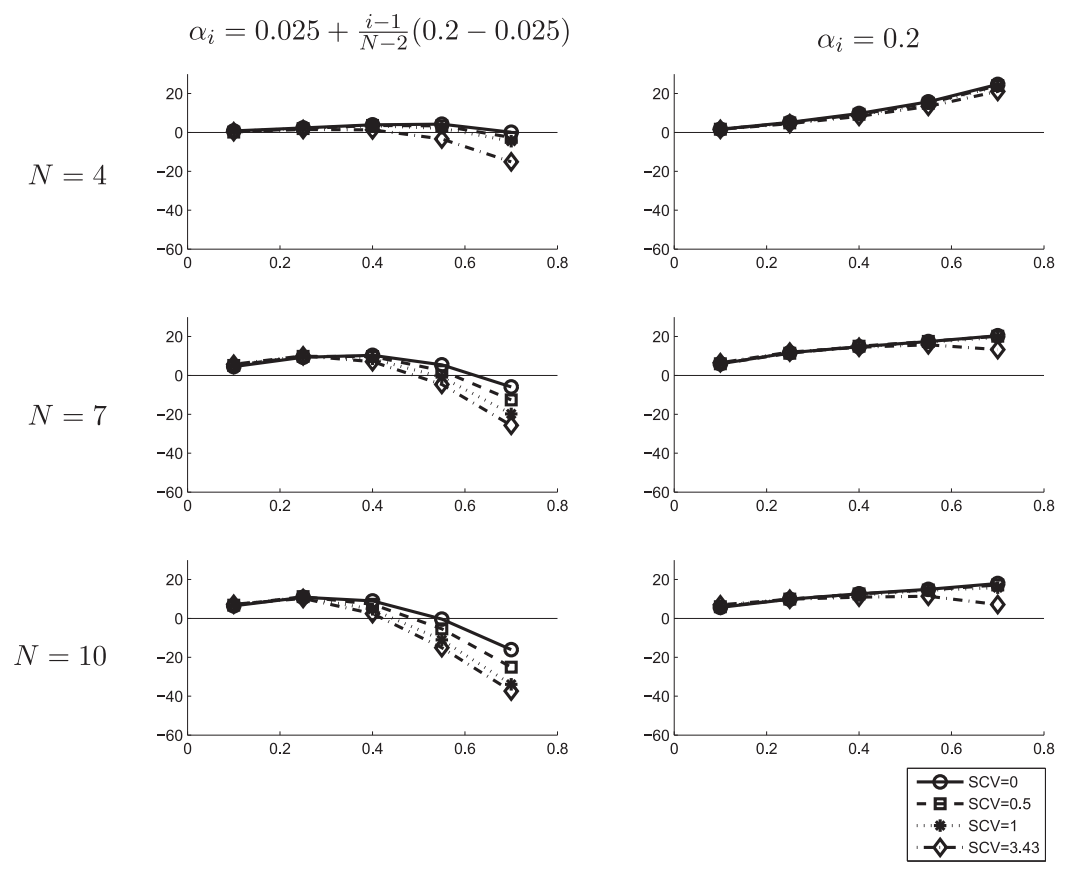

Figure 4. Relative errors $(\%)$ in the sojourn time of the last mobile node (on the vertical axis) as function of the maximal load (on the horizontal axis) for $\alpha_{i}=0.0252-$ $\frac{i-1}{N-2}(2-0.0025)$ and $\alpha_{i}=0.25$. 
(a)

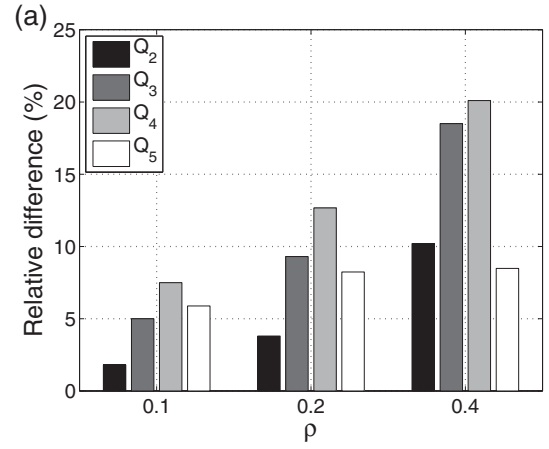

(b)

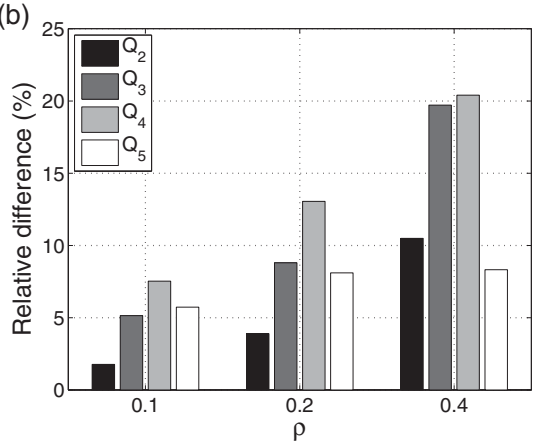

Figure 5. Relative difference between the queue-length approximation and the simulation as function of $\rho$ for $\alpha=0.05$ and $b=1$ with: (a) exponential service requirement, (b) deterministic service requirement.

measure like the total sojourn time, the absolute figures change, but the shapes of the figures only change slightly.

In the following sections, we focus on the effects of changing one parameter at a time. In these cases, we not only give the relative accuracy of our approximation in the one but last queue but also in the other queues.

\subsection{Accuracy of Queue-Length Approximation versus Load}

In this section, we study the accuracy of the mean sojourn time in $Q_{i}$ using the queuelength approximation by comparing it to the simulation results as function of the queue load $\rho_{i}$. This will be done for both the symmetric case when $\alpha_{i}=\alpha$ for $i=2, \ldots, N-1$, and asymmetric case when $\alpha_{i} \neq \alpha_{j}$ for some $i$ and $j$.

6.2.1. Symmetric case. We consider a tandem network of six queues, that is, $N=6$, with mean service time $b=1$ and $\alpha_{i}=0.05, i=2, \ldots, 5$. Note that in the case of exponential services the load at the queues satisfies $\rho_{2}=\rho_{3}=\rho_{4}=2 \rho_{5}=\rho$. However, in the case of deterministic or hyper-exponential the load at the queues satisfies $\rho_{2}=\rho_{3}=\rho_{4}=\rho \approx 2 \rho_{5}$; see Eq. (6). Figures 5 and 6 show the relative difference as function of $\rho$ for exponential, deterministic, and hyper-exponential service distribution. Observe that $\tau_{i}^{\mathrm{ql}}$ is smaller than $20 \%$ for $\rho \leq 0.4$ and for all service distributions. For this reason, the queue-length approximation is accurate in the cases of light and moderate load at $Q_{i}$. Moreover, we note that the accuracy of the approximation is almost the same for the considered service distributions.

6.2.2. Asymmetric case. We consider a tandem network with $N=6$ queues including source and destination. Our objective is to show that the approximated and simulated mean sojourn time follow the same pattern for $i=2, \ldots, 6$. We consider two different settings for $\left\{\alpha_{2}, \ldots, \alpha_{5}\right\}$ : the allocation set $A=\{0.05,0.025,0.1,0.0375,0.05\}$ and the set $B=\{0.05,0.1,0.15,0.2,0.05\}$. Figure 7 displays the mean sojourn time at $Q_{i}$ as function of $\alpha_{i}$ for exponential service requirement. Observe that the approximation predicts the behavior of the simulation very well. Moreover, the queues with the highest and lowest mean sojourn time are the same in the simulation and approximation. These observations also hold for the hyper-exponential service distribution as shown in Figure 8. 

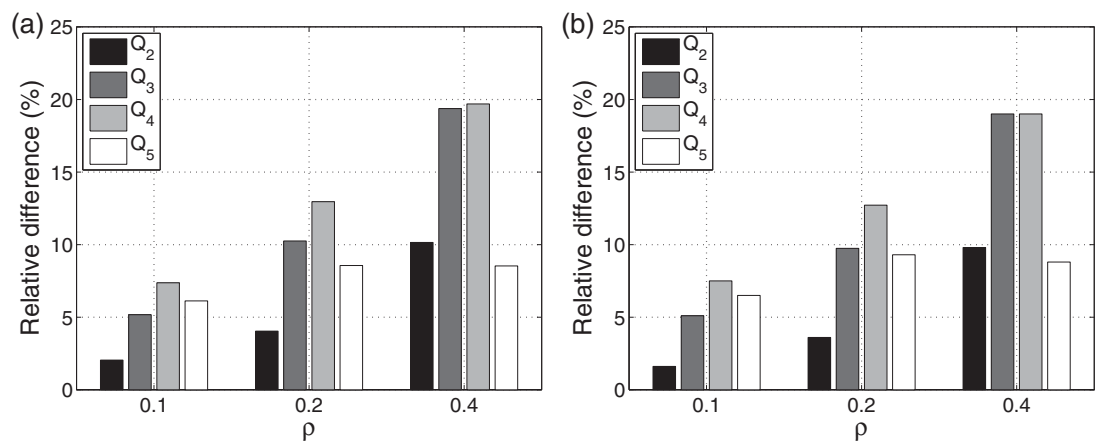

Figure 6. Relative difference between the approximation and the simulation as function of $\rho$ for $\alpha=0.05$ and $b=1$ with: (a) hyper-exponential service time with $p_{1}=0.6$, $m_{1}=0.1$, and $\mathrm{SCV}=3.43$, (b) hyper-exponential service time with $p_{1}=0.8, m_{1}=0.1$, and $\mathrm{SCV}=7.48$.

(a)

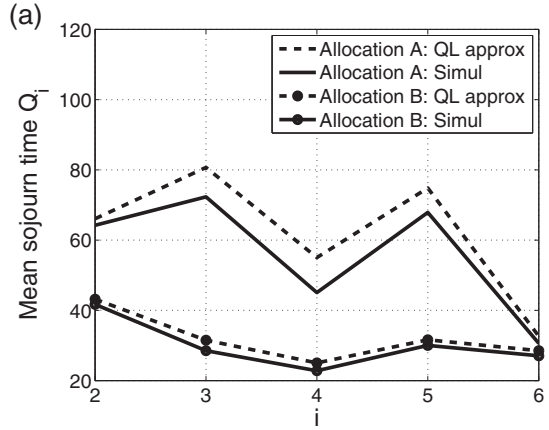

(b)

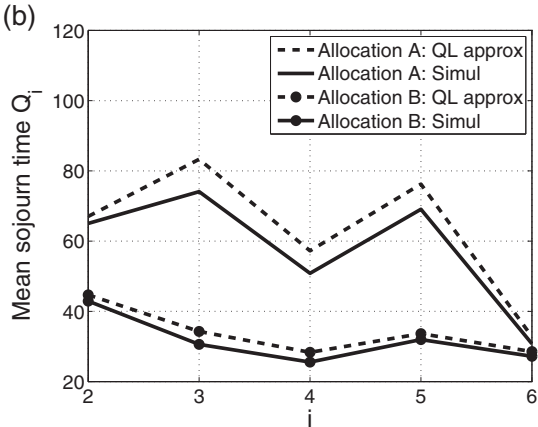

Figure 7. Mean sojourn time at $Q_{i}$ using queue-length approximation and simulation for $\lambda=0.05$ and $b=1$ with: (a) exponential service time, (b) deterministic service time.
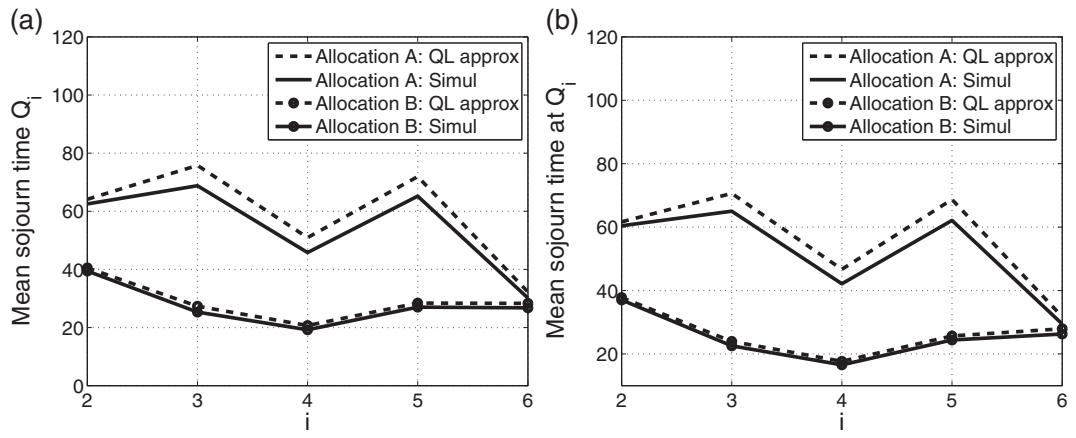

Figure 8. Mean sojourn time at $Q_{i}$ using queue-length approximation and simulation for $\lambda=0.05$ and $b=1$ with: (a) hyper-exponential service time with $p_{1}=0.6$ and $m_{1}=0.1$, and $\mathrm{SCV}=3.43$, (b) hyper-exponential service time with $p_{1}=0.8, m_{1}=0.1$, and $\mathrm{SCV}=7.48$. 

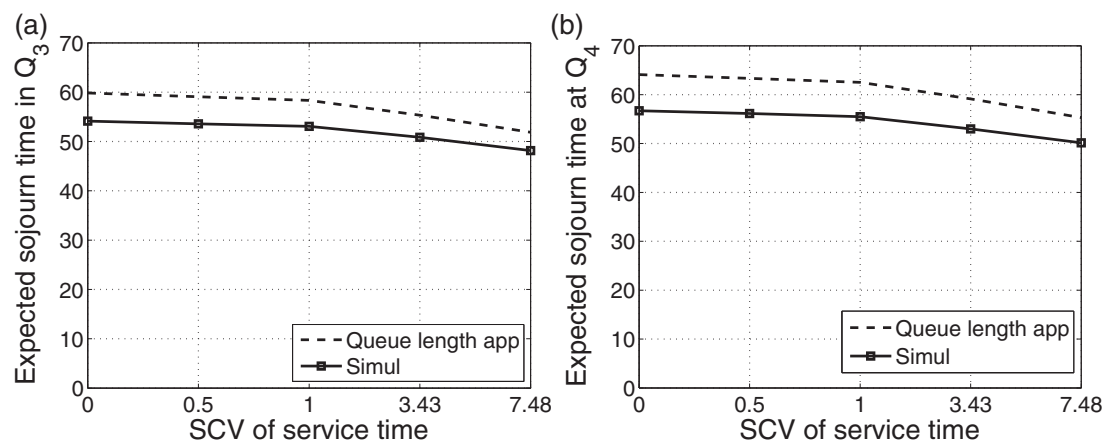

Figure 9. Expected sojourn time at $Q_{3}$ and $Q_{4}$ as function of the SCV of the service times for $\alpha=0.05$ and $\lambda=0.05$.

TABle 1. Coefficient of variation and second moment of the sojourn time at $Q_{i}$ using queue-length and workload approximation and simulation for: $\lambda=0.05$, exponential service with $b=1$, for the $\alpha_{i}$ allocation set $A$ (Top) and $B$ (bottom)

\begin{tabular}{lccccc}
\hline & $Q_{2}$ & $Q_{3}$ & $Q_{4}$ & $Q_{5}$ & $Q_{6}$ \\
\hline$\alpha_{i}$ & 0.05 & 0.1 & 0.15 & 0.2 & 0.05 \\
$\sigma_{i}^{\mathrm{ql}}$ & 0.785 & 0.811 & 0.830 & 1.084 & 0.791 \\
$\sigma_{i}^{\text {sim }}$ & 0.778 & 0.782 & 0.795 & 1.099 & 0.801 \\
$\mathbb{E}\left[\left(D_{i}^{\mathrm{ql}}\right)^{2}\right]$ & 3328.0 & 1796.4 & 1147.9 & 2080.9 & 1453.0 \\
$\mathbb{E}\left[\left(D_{i}^{\text {sim }}\right)^{2}\right]$ & 3094.0 & 1449.8 & 933.9 & 1891.0 & 1318.8 \\
$\alpha_{i}$ & 0.05 & 0.025 & 0.1 & 0.0375 & 0.05 \\
$\sigma_{i}^{\mathrm{ql}}$ & 0.998 & 0.761 & 0.981 & 0.815 & 0.761 \\
$\sigma_{i}^{\operatorname{sim}}$ & 1.022 & 0.741 & 0.985 & 0.795 & 0.756 \\
$\mathbb{E}\left[\left(D_{i}^{\mathrm{ql}}\right)^{2}\right]$ & 8726.9 & 11466.6 & 6000.3 & 10163.8 & 1875.6 \\
$\mathbb{E}\left[\left(D_{i}^{\text {sim }}\right)^{2}\right]$ & 8338.7 & 9098.3 & 4780.2 & 8266.8 & 1643.7 \\
\hline
\end{tabular}

\subsection{Mean Approximate Sojourn Time versus Service Times Distribution}

Let us check the behavior of the queue-length approximation as function of the service times distribution. We consider the symmetric scenario of a tandem network of six queues, that is, $N=6$, with mean service requirement $b=1$ and $\alpha_{i}=0.05, i=2, \ldots, 5$. Figure 9 displays the expected sojourn time at $Q_{3}$ and $Q_{4}$ using the approximation as function of the SCV of the service times. For $\lambda=0.05$ and $\lambda=0.1$, respectively, observe that the accuracy of the queue-length approximation is almost insensitive of the SCV of the service times. Furthermore, for all parameter values considered the approximated mean delay in $Q_{i}$ gives an upper bound of the simulated mean delay in $Q_{i}$. This observation is in support of the result in [4] which proves that in the correlated $M / G / 1$ queue a positive correlation between the service time and the last inter-arrival time reduces the mean sojourn time. We should emphasize that in our model $K_{i, n}^{m}$ and the last inter-arrival time are positively correlated, that is, an increase of the last inter-arrival time induces stochastically an increase of $K_{i, n}^{m}$. 
TABle 2. Coefficient of variation and second moment of the sojourn time at $Q_{i}$ using queue-length and workload approximation and simulation for: $\lambda=0.05$, deterministic service with $b=1$, for the $\alpha_{i}$ allocation set $A$ (Top) and $B$ (bottom)

\begin{tabular}{lccccc}
\hline & $Q_{2}$ & $Q_{3}$ & $Q_{4}$ & $Q_{5}$ & $Q_{6}$ \\
\hline$\alpha_{i}$ & 0.05 & 0.1 & 0.15 & 0.2 & 0.05 \\
$\sigma_{i}^{\mathrm{ql}}$ & 0.775 & 0.799 & 0.817 & 1.052 & 0.780 \\
$\sigma_{i}^{\text {sim }}$ & 0.767 & 0.763 & 0.769 & 1.060 & 0.791 \\
$\mathbb{E}\left[\left(D_{i}^{\mathrm{ql}}\right)^{2}\right]$ & 3542.7 & 2112.5 & 1465.4 & 2322.6 & 1449.5 \\
$\mathbb{E}\left[\left(D_{i}^{\text {sim }}\right)^{2}\right]$ & 3254.1 & 1650.2 & 1155.2 & 2102.5 & 1324.8 \\
$\alpha_{i}$ & 0.05 & 0.025 & 0.1 & 0.0375 & 0.05 \\
$\sigma_{i}^{\mathrm{ql}}$ & 0.99 & 0.755 & 0.968 & 0.809 & 0.748 \\
$\sigma_{i}^{\text {sim }}$ & 1.012 & 0.734 & 0.964 & 0.787 & 0.742 \\
$\mathbb{E}\left[\left(D_{i}^{\mathrm{ql}}\right)^{2}\right]$ & 8941.5 & 12182.8 & 6441.7 & 10513.9 & 1882.0 \\
$\mathbb{E}\left[\left(D_{i}^{\text {sim }}\right)^{2}\right]$ & 8511.1 & 9513.6 & 5075.6 & 8650.5 & 1650.1 \\
\hline
\end{tabular}

TABle 3. Coefficient of variation and second moment of the sojourn time at $Q_{i}$ using queue-length and workload approximation and simulation for: $\lambda=0.05$, hyper-exponential service with $b=1, p_{1}=0.6$, and $m_{1}=0.1$, for the $\alpha_{i}$ allocation set $A$ (Top) and $B$ (bottom)

\begin{tabular}{lccccc}
\hline & $Q_{2}$ & $Q_{3}$ & $Q_{4}$ & $Q_{5}$ & $Q_{6}$ \\
\hline$\alpha_{i}$ & 0.05 & 0.1 & 0.15 & 0.2 & 0.05 \\
$\sigma_{i}^{\mathrm{ql}}$ & 0.805 & 0.833 & 0.854 & 1.142 & 0.816 \\
$\sigma_{i}^{\text {sim }}$ & 0.897 & 0.903 & 0.912 & 1.081 & 0.907 \\
$\mathbb{E}\left[\left(D_{i}^{\mathrm{ql}}\right)^{2}\right]$ & 2958.7 & 1368.5 & 793.9 & 1729.5 & 1456.0 \\
$\mathbb{E}\left[\left(D_{i}^{\text {sim }}\right)^{2}\right]$ & 2805.4 & 1162.1 & 675.9 & 1582.4 & 1306.1 \\
$\alpha_{i}$ & 0.05 & 0.025 & 0.1 & 0.0375 & 0.05 \\
$\sigma_{i}^{\mathrm{ql}}$ & 1.015 & 0.773 & 1.010 & 0.826 & 0.789 \\
$\sigma_{i}^{\text {sim }}$ & 1.018 & 0.871 & 1.011 & 0.899 & 0.885 \\
$\mathbb{E}\left[\left(D_{i}^{\mathrm{ql}}\right)^{2}\right]$ & 8288.3 & 10180.8 & 5214.7 & 9449.5 & 1857.9 \\
$\mathbb{E}\left[\left(D_{i}^{\text {sim }}\right)^{2}\right]$ & 7956.4 & 8321.6 & 4241.5 & 7687.5 & 1620.7 \\
\hline
\end{tabular}

\subsection{SCV of Sojourn Time in $Q_{i}$}

Next, we compare the SCV $\sigma_{i}$ of the sojourn time at $Q_{i}, \sigma_{i}:=\operatorname{Var}\left[D_{i}\right] / \mathbb{E}\left[D_{i}\right]^{2}$, following from the queue-length approximations with the simulation denoted as $\sigma_{i}^{\mathrm{ql}}$ and $\sigma_{i}^{\mathrm{sim}}$, respectively. Tables $1-3$ show $\sigma_{i}^{\mathrm{ql}}$ and $\sigma_{i}^{\text {sim }}$, and also the second moments $\mathbb{E}\left[\left(D_{i}^{\mathrm{ql}}\right)^{2}\right]$ and $\mathbb{E}\left[\left(D_{i}^{\mathrm{sim}}\right)^{2}\right]$ for the exponential, the deterministic, and the hyper-exponential service times distribution. Observe that the SCV of the approximations are accurate. 
TABLE 4. Mean sojourn time at $Q_{i}$ using the queue-length approximation as function of $\alpha$ for: $\lambda=0.075$, exponential service with $b=1$

\begin{tabular}{lrrrrrr}
\hline$\alpha$ & $\rho_{2}$ & $Q_{2}$ & $Q_{3}$ & $Q_{4}$ & $Q_{5}$ & \multicolumn{1}{c}{$Q_{6}$} \\
\hline 0.05 & 0.3 & 60.55 & 71.64 & 76.54 & 78.14 & 35.62 \\
0.1 & 0.3 & 33.23 & 38.04 & 41.43 & 42.42 & 19.01 \\
0.2 & 0.3 & 19.55 & 22.25 & 23.36 & 23.75 & 10.75 \\
0.4 & 0.3 & 12.69 & 13.96 & 14.39 & 14.52 & 6.34 \\
0.8 & 0.3 & 9.24 & 9.82 & 9.96 & 10.00 & 4.29 \\
50 & 0.3 & 5.77 & 5.78 & 5.78 & 5.79 & 2.38 \\
\hline
\end{tabular}

TABLE 5. Mean sojourn time at $Q_{i}$ using the queue-length approximation as function of $\alpha$ for: $\lambda=0.075$, deterministic service with $b=1$

\begin{tabular}{llccccc}
\hline$\alpha$ & $\rho_{2}$ & $Q_{2}$ & $Q_{3}$ & $Q_{4}$ & $Q_{5}$ & $Q_{6}$ \\
\hline 0.05 & 0.315 & 62.49 & 74.37 & 23.52 & 20.95 & 22.99 \\
0.1 & 0.33 & 35.77 & 42.21 & 44.94 & 45.91 & 19.34 \\
0.2 & 0.37 & 23.52 & 27.10 & 28.31 & 28.67 & 11.03 \\
0.4 & 0.46 & 20.98 & 23.30 & 23.79 & 23.90 & 7.24 \\
0.8 & 0.74 & 49.57 & 53.01 & 53.37 & 53.58 & 6.26 \\
\hline
\end{tabular}

\subsection{Impact of $\alpha_{i}$ on Mean Sojourn Time}

Our objective is to show the impact of $\alpha_{i}$ on the mean sojourn time at $Q_{i}$ as function of the service time distribution. We consider the symmetric case where $\alpha_{i}=\alpha$. Table 4 shows the mean sojourn time at $Q_{i}$ as function of $\alpha$ in the case of exponential service times. Note that for $\lambda=0.075$ and $b=1$, the load $\rho_{i}, i=2, \ldots, 5$, is equal to 0.3 and $\rho_{6}=0.15$. Observe that the mean sojourn time decreases at $Q_{i}$ with $\alpha$. Moreover, the mean sojourn time at $Q_{i}, i=2, \ldots, 5$, converges to the mean sojourn time in an $\mathrm{M} / \mathrm{M} / 1$ queue with load 0.3 and arrival rate $\lambda=0.075$ that is equal to 5.71. A similar result holds for $Q_{6}$ which gives that its limiting mean sojourn time is equal to 2.38 . Table 5 displays the mean sojourn time at $Q_{i}$ as function of $\alpha$ in the case of deterministic service. The mean sojourn time of the deterministic service as function of $\alpha$ has an optimum value for $\alpha$. Additional experiments show that this optimum is around 0.4. The hyper-exponential service gives similar results as the case of exponential service (see Table 6). That is, the mean sojourn time in $Q_{i}$, $i=2, \ldots, 5$, is decreasing with $\alpha$ and it converges to a limit value, which is approximately equal to the mean sojourn time in an $\mathrm{M} / \mathrm{M} / 1$ queue with arrival rate $\lambda$ and load $\rho_{i}$. For the deterministic service, we note that the optimal value of $\alpha$ is sensitive to the value of $\lambda$ and $b$ in such a way that the higher the load at the queue the smaller the optimal value of $\alpha$.

\section{CONCLUSION AND POSSIBLE GENERALIZATION}

In this paper, we have addressed the performance of a tandem queueing system with mobile queues. We have proposed an analytical approximation for the LST of the delay in $Q_{i}$. The approximation is called queue-length approximation. Through extensive numerical validation we have shown that the queue-length approximation gives nice results for light and moderate load in the case of general service times distribution. 
TABLE 6. Mean sojourn time at $Q_{i}$ using the queue-length approximation as function of $\alpha$ for: $\lambda=0.075$, hyper-exponential service with $b=1, p_{1}=0.6$, and $m_{1}=0.1$

\begin{tabular}{llccccc}
\hline$\alpha$ & $\rho_{2}$ & $Q_{2}$ & $Q_{3}$ & $Q_{4}$ & $Q_{5}$ & $Q_{6}$ \\
\hline 0.05 & 0.27 & 56.83 & 66.39 & 70.96 & 72.58 & 35.00 \\
0.1 & 0.24 & 29.29 & 33.58 & 35.87 & 36.89 & 18.31 \\
0.2 & 0.21 & 15.33 & 17.05 & 17.97 & 18.39 & 9.64 \\
0.4 & 0.16 & 8.19 & 8.82 & 9.15 & 9.31 & 5.2 \\
0.8 & 0.124 & 4.5 & 4.73 & 4.84 & 4.89 & 2.88 \\
50 & 0.05 & 0.74 & 0.74 & 0.75 & 0.75 & 0.38 \\
\hline
\end{tabular}

For the sake of clarity, we restricted ourselves to the case where the switch-over time, that is the time needed for a queue to alternate between locations, is equal to zero. As a generalization, we can assume that the switch-over time distribution is phase-type, which increases the cardinality of the state space. For example, in Section 5.2, assume that when $Q_{i}$ migrates from location $L_{i}$ to $L_{i-1}$ it requires an exponentially distributed switch-over time with mean $c_{i}^{-}$. Similarly, when $Q_{i}$ migrates from location $L_{i-1}$ to $L_{i}$ it requires an exponentially distributed switch-over time with mean $c_{i}^{+}$. The state space of the Markov chain $\left(L_{i-1}(t), L_{i}(t), L_{i+1}(t)\right)$ is equal to $\Omega=\{-2,-1,0,1\}^{3}$, where $L_{i}(t)=-1$ $\left(L_{i}(t)=-2\right)$ when $Q_{i}$ switches from $L_{i-1}$ to $L_{i}\left(L_{i}\right.$ to $\left.L_{i-1}\right)$. Following the footprints of Section 5.2 , one can easily show that the $\hat{M}_{n}(z)$ has exactly the same form as depicted in Lemmas 2 and 3. The matrices $\mathbf{A}$ and $\mathbf{B}$ in this case have a much larger dimension. More precisely, $\mathbf{A}$ (resp. $\mathbf{B}$ and $\mathbf{H}$ ) is a 60 -by-60 matrix.

In this paper, we restricted ourselves to the Tandem model case. The case of a general network of queues with fork and join traffic and with mobile queues remains an open problem to be addressed in the future. Moreover, we considered the case where there is a single mobile node moving between two consecutive locations. The scenario of multiple mobile nodes moving between two consecutive locations is important to address some applications such as in vehicular networks where for example the mobile nodes represent the busses moving between to stations.

\section{Acknowledgements}

In the Netherlands, the three Universities of Technology have formed the 3TU.Federation. This article is the result of joint research in the 3TU.Centre of Competence NIRICT (Netherlands Institute for Research on ICT). The authors acknowledge De Nederlandse Organisatie voor Wetenschappelijk Onderzoek (NWO) for their financial support.

\section{References}

1. Al Hanbali, A., de Haan, R., Boucherie, R.J. \& van Ommeren, J.K. (2008). A tandem queueing model for delay analysis in disconnected ad hoc networks. Proc. ASMTA, LCNS 5055, Nicosia, Cyprus, pp. 189-205

2. Al Hanbali, A., de Haan, R., Boucherie, R.J. \& van Ommeren, J.K. (2012). Time-limited polling systems with batch arrivals and phase-type service times. Annals of Operations Research 198: 57-82

3. Al Hanbali, A., Mandjes, M., Nazarathy, Y. \& Whitt, W. (2011). The asymptotic variance of departures in critically loaded queues. Advances in Applied Probability 43: 243-263

4. Borst, S., Boxma, O. \& Combé, M. (1992). Collection of customers: a correlated $M / G / 1$ queue. Performance Evaluation 20: 47-59 
5. Coffman, E.G., Fayolle, G. \& Mitrani, I. (1988). Two queues with alternating service periods. In: Performance '87: Proc. of the 12th IFIP WG 7.3 Int. Symp. Computer Performance Modelling, Measurement and Evaluation. pp. 227-239

6. de Haan, R., Al Hanbali, A., Boucherie, R.J. \& van Ommeren, J.K. (2009). A transient analysis of polling systems operating under exponential time-limited service disciplines. Research Memorandum 1894, University of Twente, Netherlands

7. de Haan, R., Boucherie, R.J. \& van Ommeren, J.K. (2009). A polling model with an autonomous server. Queueing Systems 62: 279-308

8. Delay Tolerant Networking Research Group, Web site: http://www.dtnrg.org.

9. Doshi, B. (1986). Queueing systems with vacations-a survey. Queueing Systems 1: 29-66

10. Eliazar, I. \& Yechiali, U. (1998). Polling under the randomly-timed gated regime. Stochastic Models 14: 79-93

11. Frigui, I. \& Alfa, A. (1998). Analysis of a time-limited polling system. Computer Communications 21(6): 558-571

12. Grossglauser, M. \& Tse, D. (2002). Mobility increases the capacity of ad hoc wireless networks. IEEE Transactions on Networking 10: 477-486

13. Leung, K. (1994). Cyclic-service systems with non-preemptive time-limited service. IEEE Transactions on Communications 42: 2521-2524

14. Neuts, M. (1981). Matrix-Geometric Solutions in Stochastic Models: An Algorithmic Approach. Baltimore: Johns Hopkins University Press

15. Katayama, T. (2001). Waiting time analysis for a queueing system with time-limited service and exponential timer. Naval Research Logistics 48: 638-651

16. van Ommeren, J.K. (1991). The discrete-time single-server queue. Queueing Systems 8: 279-294

17. van Vuuren, M., Adan, I. \& Resing-Sassen, S. (2005). Performance analysis of multi-server tandem queues with finite buffers and blocking. OR Spectrum 27: 315-338

18. Wang, C. \& Wolff, R. (2005). Work-conserving tandem queues. Queueing Systems 49: 283-296

19. Zazanis, M. (1998). A Palm calculus approach to functional versions of Little's law. Stochastic Processes and their Applications 74: 195-201

\section{APPENDIX A: PROOFS}

In this section, we will use the theory of the finite-state continuous-time absorbing Markov chains to compute $\hat{M}_{n}(z)$. Lemma 6 summarizes some known results, e.g., see [14], of this theory that will be used afterwards.

Lemma 6: Consider a finite-state, continuous-time, Markov chain $\{M C(t), t \geq 0\}$, with state space $\zeta=\{1, \ldots, m+n\}$ and with infinitesimal generator matrix, $\mathbf{G}$, of the form

$$
\mathbf{G}=\left(\begin{array}{c|c}
\mathbf{U} & \mathbf{V} \\
\hline \mathbf{0}_{m} & \mathbf{0}_{n}
\end{array}\right),
$$

where $\mathbf{U}$ is an $m$-by-m matrix, $\mathbf{V}$ is an $m$-by-n matrix, $\mathbf{0}_{m}$ is an $n$-by-m matrix with entries equal to 0 and $\mathbf{0}_{n}$ is an $n$-by-n matrix with entries equal to 0 . The states $\{m+1, \ldots, m+n\}$ are absorbing. Then,

(a) the states $\{1, \ldots, m\}$ are all transient if and only if $\mathbf{U}$ is a non-singular matrix.

(b) The probability distribution, $F($.$) , of the time until absorption in one of the absorbing states$ $\{m+1, \ldots, m+n\}$, given that $M C(0)=i, i=1, \ldots, m$, reads

$$
F(t)=1-\alpha_{i} \exp (\mathbf{U} t) e, \quad t \geq 0,
$$

where $\alpha_{i}$ is the m-dimensional row vector with entries equal to 0 except the ith one that is equal to 1 , e is the $m$-dimensional column vector with entries all equal to 1 , and where

$$
\exp (\mathbf{U} t):=\sum_{i=0}^{\infty} \frac{(\mathbf{U} t)^{i}}{i !},
$$


with $(\mathbf{U} t)^{0}=\mathbf{I}_{m}$ the $m$-by-m identity matrix. Similarly, the LST, $\tilde{F}(s)$, of the time until absorption in one of the states $\{m+1, \ldots, m+n\}$, given that $M C(0)=i, i=1, \ldots, m$, reads

$$
\tilde{F}(s)=\alpha_{i}\left(s \mathbf{I}_{m}-\mathbf{U}\right)^{-1} \mathbf{V} e_{n}, \quad \text { Re } s \geq 0,
$$

where $e_{n}$ is the $n$-dimensional column vector with entries are all equal to 1.

(c) Given that $M C(0)=i$, the expected amount of time spent in the transient state $j$ is equal to the (i,j)-entry of $-\mathbf{U}^{-1}, i, j=1, \ldots, m$.

(d) Given that $M C(0)=i$, the probability that absorption occurs in state $j$ is equal to the (i,j)-entry of $-\mathbf{U}^{-1} \mathbf{V}, i=1, \ldots, m$ and $j=m+1, \ldots, m+n$.

\section{A.1. Proof of Lemma 2}

Recall that $M_{n}^{+}$is equal to $M_{n}$ given that $M_{n}>0$, where $M_{n}$ is the total number of $Q_{i}$ arrival periods during the time interval that separates two consecutive $Q_{i}$ service periods.

Let $W(t)=\left(L_{i-1}(t), L_{i}(t), L_{i+1}(t), M(t)\right)$ denote the continuous-time Markov chain with discrete state-space $\{0,1\}^{3} \times\{1,2, \ldots\}$, where $M(t)$ is the number of $Q_{i}$ arrival periods until time $t$ given that it is strictly positive. Assume that $\left(L_{i-1}(0), L_{i}(0), L_{i+1}(0)\right)$ is in steadystate and that a $Q_{i}$ arrival period has just started at 0 , that is, time 0 is the first time that $\left(L_{i-1}(0), L_{i}(0), L_{i+1}(0)\right)=(0,1, \cdot)$ with $\left(L_{i-1}(0-), L_{i}(0-)\right) \neq(0,1)$. Moreover, we set $M(0)=1$ and $M(0-)=0$, and make the states $(\cdot, 0,1, \cdot)$ of $W(t)$ to be absorbing. Merging these absorbing states into one state, referred to as a, will not impact the dynamics of $W(t)$ before absorption. Since $\left(L_{i-1}(t), L_{i}(t), L_{i+1}(t)\right)$ is an irreducible Markov chain, the probability of transition to state $a$ is equal to one and thus the time until absorption, $T_{a}$, is a proper rv. We will refer to the previous absorbing chain as AMC. Now writing $M_{n}^{+}$in terms of $M(t)$ gives that $M_{n}^{+}=M\left(T_{a}\right)$. The probability distribution $\mathbb{P}\left(M_{n}^{+}=m\right)$ is the probability that the transition to a occurs from one of the states $\{(i, j, k, m): i, j, k=0,1$ and $(j, k) \neq(0,1)\}$.

We derive now $\hat{M}_{n}^{+}(z)$, the p.g.f. of $M_{n}^{+}$. Let us define a level $l(m), m=1,2, \ldots$, to be the transient states of AMC with $M(t)=m$ and ordered as follows

$$
l(m):=\{(0,0,0, m),(0,1,0, m),(0,1,1, m),(1,0,0, m),(1,1,0, m),(1,1,1, m)\},
$$

Observe that there are in total six states in $l(m)$. We order the infinite number of AMC states as follows: $l(1), l(2), \ldots$, and finally the absorbing state $a$. It is easily seen that the generator matrix $\mathbf{P}$ of $\mathbf{A M C}$ can be written as

$$
\mathbf{P}=\left(\begin{array}{cc}
\mathbf{Q} & \mathbf{R} \\
\mathbf{0}^{T} & 0
\end{array}\right)
$$

where $\mathbf{Q}$ represents the generator matrix of transitions between the transient states of $\mathbf{A M C}, \mathbf{R}$ represents the rate vector of transitions from the transient states to the absorbing state $a, \mathbf{0}^{T}$ is the row vector with all entries equal to zero. Let $u$ denote a column vector that designates the transition rate vector from $l(m)$ states to the state $a$. Therefore, $u=\left(\alpha_{i+1}^{0}, 0, \alpha_{i}^{1}, \alpha_{i+1}^{0}, 0, \alpha_{i}^{1}\right)^{T}$. Since $u$ is independent of $m$, the vector $\mathbf{R}^{T}=\left(u^{T}, u^{T}, \ldots\right)$. Note that on leaving $l(m)$ the AMC either jumps to $l(m+1)$ or to $a$. For this reason, $\mathbf{Q}$ is an infinite upper-bidiagonal block matrix of the following form

$$
\mathbf{Q}=\left(\begin{array}{llllcl}
\mathbf{A} & \mathbf{B} & & \mathbf{0} & \ldots & \ldots \\
\mathbf{0} & \mathbf{A} & & \mathbf{B} & \mathbf{0} & \ldots \\
\vdots & & \ddots & & \ddots & \ddots
\end{array}\right)
$$


where, $\mathbf{A}$ is a six-by-six matrix that represents the transition rates between the states of $l(m)$, $m=1,2, \ldots$, which reads

\begin{tabular}{c||cccccc|} 
& $0,0,0, m$ & $0,1,0, m$ & $0,1,1, m$ & $1,0,0, m$ & $1,1,0, m$ & $1,1,1, m$ \\
\hline \hline $0,0,0, m$ & $A_{11}$ & 0 & 0 & $\alpha_{i-1}^{0}$ & 0 & 0 \\
$0,1,0, m$ & $\alpha_{i}^{1}$ & $A_{22}$ & $\alpha_{i+1}^{0}$ & 0 & $\alpha_{i-1}^{0}$ & 0 \\
$0,1,1, m$ & 0 & $\alpha_{i+1}^{1}$ & $A_{33}$ & 0 & 0 & $\alpha_{i-1}^{0}$ \\
$1,0,0, m$ & $\alpha_{i-1}^{1}$ & 0 & 0 & $A_{44}$ & $\alpha_{i}^{0}$ & 0 \\
$1,1,0, m$ & 0 & 0 & 0 & $\alpha_{i}^{1}$ & $A_{55}$ & $\alpha_{i+1}^{0}$ \\
$1,1,1, m$ & 0 & 0 & 0 & 0 & $\alpha_{i+1}^{1}$ & $A_{66}$ \\
\hline
\end{tabular}

B is a six-by-six matrix that represents the transition rates from the states of $l(m)$ to $l(m+1)$, $m=1,2, \ldots$, which reads

\begin{tabular}{c||cccccc|} 
& $0,0,0, n$ & $0,1,0, n$ & $0,1,1, n$ & $1,0,0, n$ & $1,1,0, n$ & $1,1,1, n$ \\
\hline \hline $0,0,0, m$ & 0 & $\alpha_{i}^{0}$ & 0 & 0 & 0 & 0 \\
$0,1,0, m$ & 0 & 0 & 0 & 0 & 0 & 0 \\
$0,1,1, m$ & 0 & 0 & 0 & 0 & 0 & 0 \\
$1,0,0, m$ & 0 & 0 & 0 & 0 & 0 & 0 \\
$1,1,0, m$ & 0 & $\alpha_{i-1}^{1}$ & 0 & 0 & 0 & 0 \\
$1,1,1, m$ & 0 & 0 & $\alpha_{i-1}^{1}$ & 0 & 0 & 0 \\
\hline
\end{tabular}

where $n=m+1$. The diagonal entries of $\mathbf{A}$ are such that $(\mathbf{A}+\mathbf{B}) e+u=\mathbf{0}$, where $e$ is the column vector of order six and with all entries equal to 1 .

Next, we will derive $\mathbb{P}\left(M_{n}^{+}=m\right)$ as function of the blocks of the inverse of $\mathbf{Q}$. Since $\mathbf{Q}$ is an upper-bidiagonal block matrix, it is easily verified that $\mathbf{Q}^{-1}$ is an upper-triangular block matrix of blocks $\mathbf{U}_{l, m}=\left(-\mathbf{A}^{-1} \mathbf{B}\right)^{m-l} \mathbf{A}^{-1}$ for $l \geq 1$ and $m \geq l$. Note that the matrix $\mathbf{A}$ is invertible since it is a generator matrix of a transient chain. Moreover, $-\mathbf{A}^{-1} \mathbf{B}$ is a sub-stochastic probability matrix whose entries give the probability of jumping to level $l(m+1)$ given that the AMC starts in $l(m)$. For this reason, $\left(-\mathbf{A}^{-1} \mathbf{B}\right)^{m} \rightarrow \mathbf{0}$ as $m \rightarrow \infty$.

From the theory of absorbing Markov chains, given that AMC starts in $l(1)$ with probability distribution vector $b$, the probability that the absorption occurs from one of the states of level $l(m)$ is given by (see Lemma 6.(d))

$$
\mathbb{P}\left(M_{n}^{+}=m\right)=-b \mathbf{U}_{1, m} u=-b\left(-\mathbf{A}^{-1} \mathbf{B}\right)^{m-1} \mathbf{A}^{-1} u .
$$

The p.g.f. of $M_{n}^{+}$then reads

$$
\begin{aligned}
\hat{M}_{n}^{+}(z) & =-b z \sum_{m \geq 0}\left(-z \mathbf{A}^{-1} \mathbf{B}\right)^{m} \mathbf{A}^{-1} u \\
& =-b z(\mathbf{A}+z \mathbf{B})^{-1} u, \quad|z| \leq 1
\end{aligned}
$$

To complete the proof of Lemma 2 it remains to find $b$. We assumed that at time 0 the $Q_{i}$ arrival period has just started. This means that time 0 is the first time after $s(<0)$ that $\left(L_{i-1}(0), L_{i}(0), L_{i+1}(0)\right)=(0,1, \cdot)$ and $\left(L_{i-1}(s), L_{i}(s), L_{i+1}(s)\right) \neq(0,1, \cdot)$. More specifically, given that $\left(L_{i-1}(s), L_{i}(s), L_{i+1}(s)\right)$ starts in $\{(0,0,1),(1,0,1)\}$ with steady-state distribution, the process $\left(L_{i-1}(t), L_{i}(t), L_{i+1}(t)\right), s<t \leq 0$, either jumps first into $\{(0,1,0),(0,1,1)\}$, or first into $\{(0,0,0),(1,0,0),(1,1,0),(1,1,1)\}$ and later on into $\{(0,1,0),(0,1,1)\}$; see Figure A.1. Given that $\left(L_{i-1}(s), L_{i}(s), L_{i+1}(s)\right)=(\cdot, 0,1)$ with steady-state distribution, the former event occurs with a probability vector that is equal to the probability of transition to $\{(0,1,0),(0,1,1)\}$, considered as 


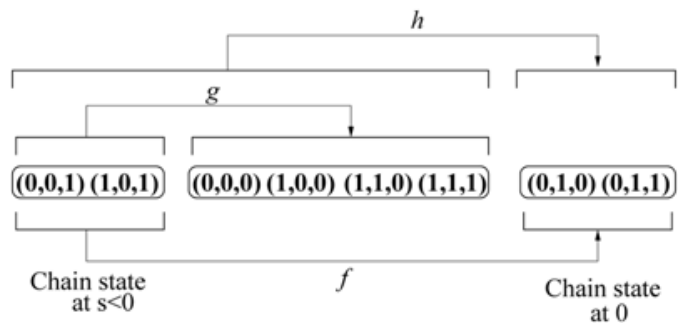

Figure A.1. Initial probability distribution of AMC that is equal to $f+h$.

absorbing set, that reads (see Lemma 6.(d))

$$
\begin{aligned}
f & =-\frac{\left(\alpha_{i-1}^{1}, \alpha_{i-1}^{0}\right)}{\alpha_{i-1}^{0}+\alpha_{i-1}^{1}}\left(\begin{array}{cc}
-\alpha_{i-1}^{0}-\alpha_{i}^{0}-\alpha_{i+1}^{1} & \alpha_{i-1}^{0} \\
\alpha_{i-1}^{1} & -\alpha_{i-1}^{1}-\alpha_{i}^{0}-\alpha_{i+1}^{1}
\end{array}\right)^{-1}\left(\begin{array}{cc}
0 & \alpha_{i}^{0} \\
0 & 0
\end{array}\right) \\
& =\frac{\left(0, \alpha_{i-1}^{1} \alpha_{i}^{0}\right)}{\left(\alpha_{i-1}^{0}+\alpha_{i-1}^{1}\right)\left(\alpha_{i}^{0}+\alpha_{i+1}^{1}\right)} .
\end{aligned}
$$

Given that $\left(L_{i-1}(s), L_{i}(s), L_{i+1}(s)\right)=(\cdot, 0,1)$, the latter event is composed of two consecutive steps: the first one occurs when the process $\left(L_{i-1}(t), L_{i}(t), L_{i+1}(t)\right)$ jumps first into $\{(0,0,0),(1,0,0),(1,1,0),(1,1,1)\}$ and the second one occurs when it jumps into $\{(0,1,0),(0,1,1)\}$; see Figure A.1. The probability vector of the first step is equal to $g$; see Eq. (A.12). For the second step, given that the process $\left(L_{i-1}(t), L_{i}(t), L_{i+1}(t)\right)$ starts in $\{(0,0,0),(1,0,0),(1,1,0),(1,1,1)\}$ with probability $g$, it is possible that the process visits $\{(0,0,1),(1,0,1)\}$ several times before it first jumps into $\{(0,1,0),(0,1,1)\}$. This occurs with probability (see Lemma $6 .(d)$ )

$$
h=-\frac{\left(\alpha_{i-1}^{1} \alpha_{i+1}^{1}, 0, \alpha_{i-1}^{0} \alpha_{i+1}^{1}, 0,0, \alpha_{i-1}^{0} \alpha_{i}^{0}\right)}{\left(\alpha_{i-1}^{0}+\alpha_{i-1}^{1}\right)\left(\alpha_{i}^{0}+\alpha_{i+1}^{1}\right)} \cdot \mathbf{H}^{-1} \cdot \mathbf{V},
$$

where,

$$
\mathbf{H}=\left(\begin{array}{cccccc}
H_{11} & \alpha_{i+1}^{0} & \alpha_{i-1}^{0} & 0 & 0 & 0 \\
\alpha_{i+1}^{1} & H_{22} & 0 & \alpha_{i-1}^{0} & 0 & 0 \\
\alpha_{i-1}^{1} & 0 & H_{33} & \alpha_{i+1}^{0} & \alpha_{i}^{0} & 0 \\
0 & \alpha_{i-1}^{1} & \alpha_{i+1}^{1} & H_{44} & 0 & \alpha_{i}^{0} \\
0 & 0 & \alpha_{i}^{1} & 0 & H_{55} & \alpha_{i+1}^{0} \\
0 & 0 & 0 & \alpha_{i}^{1} & \alpha_{i+1}^{1} & H_{66}
\end{array}\right), \mathbf{V}=\left(\begin{array}{cc}
\alpha_{i}^{0} & 0 \\
0 & \alpha_{i}^{0} \\
0 & 0 \\
0 & 0 \\
\alpha_{i-1}^{1} & 0 \\
0 & \alpha_{i-1}^{1}
\end{array}\right)
$$

and the diagonal entries of $\mathbf{H}$ are such that $\mathbf{H} e+\mathbf{V}(1,1)^{T}=\mathbf{0}$. Finally, $f+h$ gives the probability distribution of $\{(0,1,0),(0,1,1)\}$ at time 0 . Therefore, the non-zero entries of $b$ read

$$
b(0,1,0)=(f+h)(1)=h(1), \quad b(0,1,1)=(f+h)(2)=1-h(1),
$$

because, specifically, $f(1)=0$ (see also (A.6) and (A.7)). This completes the proof.

\section{A.2. Proof of Lemma 3}

The probability $\mathbb{P}\left(M_{n}=0\right)$ is the probability that no $Q_{i}$ arrival period occurs during the $n$th cycle. This happens when no $Q_{i-1}$ service period occurs between the $n$th and $(n+1)$-st $Q_{i}$ service periods. In terms of the Markov chain $\left(L_{i-1}(t), L_{i}(t), L_{i+1}(t)\right)$, the probability of the latter event reduces 
to the probability that the chain first visits the set $\{(0,0,1),(1,0,1)\}$ and later on $\{(0,1,0),(0$, $1,1)\}$, given the initial conditions that

$$
\begin{aligned}
\left(L_{i-1}(0-), L_{i}(0-), L_{i+1}(0-)\right) & \in\{(0,0,1),(1,0,1)\}, \\
\left(L_{i-1}(0), L_{i}(0), L_{i+1}(0)\right) & \in\{(0,0,0),(1,0,0),(1,1,0),(1,1,1)\} .
\end{aligned}
$$

Making the sets $\{(0,0,1),(1,0,1)\}$ and $\{(0,1,0),(0,1,1)\}$ absorbing, $\mathbb{P}\left(M_{n}=0\right)$ is the probability of absorption in $\{(0,0,1),(1,0,1)\}$ given the conditions in (A.10) and (A.11). First, let us derive the initial probability vector of the absorbing Markov chain. This initial probability vector is equal to the probability that the process jumps into $\{(0,0,0),(1,0,0),(1,1,0),(1,1,1)\}$, given that at initial time this process starts with the steady state distribution in $\{(0,0,1),(1,0,1)\}$, which can be written as (see Lemma 6.(d))

$$
\begin{aligned}
g & =-\frac{\left(\alpha_{i-1}^{1}, \alpha_{i-1}^{0}\right)}{\alpha_{i-1}^{0}+\alpha_{i-1}^{1}}\left(\begin{array}{cc}
-\alpha_{i-1}^{0}-\alpha_{i}^{0}-\alpha_{i+1}^{1} & \alpha_{i-1}^{0} \\
\alpha_{i-1}^{1} & -\alpha_{i-1}^{1}-\alpha_{i}^{0}-\alpha_{i+1}^{1}
\end{array}\right)^{-1}\left(\begin{array}{cccc}
\alpha_{i+1}^{1} & 0 & 0 & 0 \\
0 & \alpha_{i+1}^{1} & 0 & \alpha_{i}^{0}
\end{array}\right) \\
& =\frac{\left(\alpha_{i-1}^{1} \alpha_{i+1}^{1}, \alpha_{i-1}^{0} \alpha_{i+1}^{1}, 0, \alpha_{i-1}^{0} \alpha_{i}^{0}\right)}{\left(\alpha_{i-1}^{0}+\alpha_{i-1}^{1}\right)\left(\alpha_{i}^{0}+\alpha_{i+1}^{1}\right)} .
\end{aligned}
$$

It then follows from absorbing Markov chain analysis that (see Lemma 6.(d))

$$
\mathbb{P}\left(M_{n}=0\right)=-g \cdot \mathbf{F}^{-1} \cdot w,
$$

where,

$$
\mathbf{F}=\left(\begin{array}{cccc}
F_{11} & \alpha_{i-1}^{0} & 0 & 0 \\
\alpha_{i-1}^{1} & F_{22} & \alpha_{i}^{0} & 0 \\
0 & \alpha_{i}^{1} & F_{33} & \alpha_{i+1}^{0} \\
0 & 0 & \alpha_{i+1}^{1} & F_{44}
\end{array}\right), \quad w=\left(\begin{array}{c}
\alpha_{i+1}^{0} \\
\alpha_{i+1}^{0} \\
0 \\
\alpha_{i}^{1}
\end{array}\right)
$$

and where the diagonal entries of $\mathbf{F}$ are such that

$$
\mathbf{F} e+w+\left(\alpha_{i}^{0}, 0, \alpha_{i-1}^{1}, \alpha_{i-1}^{1}\right)^{T}=\mathbf{0},
$$

which completes the proof.

\section{A.3. Proof of Lemma 4}

$\Xi_{i}$ is the duration of service interruption in $Q_{i}$. Therefore, in terms of the Markov chain $\left(L_{i-1}(t), L_{i}(t), L_{i+1}(t)\right), \Xi_{i}$ is the return time of the Markov chain $\left(L_{i-1}(t), L_{i}(t), L_{i+1}(t)\right)$ to the set $\{(0,0,1),(1,0,1)\}$, given that the chain has just left this set at initial time. Let $y$ denote the row vector that represents the probability distribution of the states $\{(0,0,0),(0,1,0),(0,1,1),(1,0,0)$, $(1,1,0),(1,1,1)\}$ at the initial time. Hence, $y$ can be written as (see Lemma 6.(d))

$$
\begin{aligned}
& y=-\frac{\left(\alpha_{i-1}^{1}, \alpha_{i-1}^{0}\right)}{\alpha_{i-1}^{0}+\alpha_{i-1}^{1}}\left(\begin{array}{cc}
-\alpha_{i-1}^{0}-\alpha_{i}^{0}-\alpha_{i+1}^{1} & \alpha_{i-1}^{0} \\
\alpha_{i-1}^{1} & -\alpha_{i-1}^{1}-\alpha_{i}^{0}-\alpha_{i+1}^{1}
\end{array}\right)^{-1} \\
& \times\left(\begin{array}{cccccc}
\alpha_{i+1}^{1} & 0 & \alpha_{i}^{0} & 0 & 0 & 0 \\
0 & 0 & 0 & \alpha_{i+1}^{1} & 0 & \alpha_{i}^{0}
\end{array}\right) \\
& =\frac{\left(\alpha_{i-1}^{1} \alpha_{i+1}^{0}, 0, \alpha_{i-1}^{1} \alpha_{i}^{0}, \alpha_{i-1}^{0} \alpha_{i+1}^{1}, 0, \alpha_{i-1}^{0} \alpha_{i}^{0}\right)}{\left(\alpha_{i-1}^{0}+\alpha_{i-1}^{1}\right)\left(\alpha_{i}^{0}+\alpha_{i+1}^{1}\right)} \text {. }
\end{aligned}
$$

Considering the set $\{(0,0,1),(1,0,1)\}$ as an absorbing set, $\tilde{\Xi}_{i}(s)$ becomes the LST of the time to absorption of $\left(L_{i-1}(t), L_{i}(t), L_{i+1}(t)\right)$ with generator matrix between transient states $\mathbf{A}+\mathbf{B}$, given in Section 5.2, $u$ transition rate column vector from transient states to the absorbing set, and with initial probability distribution $y$. Lemma 6 .(b) gives the desired result of $\tilde{\Xi}_{i}(s)$. 


\section{A.4 Proof of Lemma 5}

$\Xi_{i}^{*}$ is the first time after $t$ that the server returns to $Q_{i}$ given that an arrival to $Q_{i}$ occurs at $t$. Therefore, in terms of the Markov chain $\left(L_{i-1}(t), L_{i}(t), L_{i+1}(t)\right)$ the duration of $\Xi_{i}^{*}$ is equal to the first passage time of the Markov chain $\left(L_{i-1}(t), L_{i}(t), L_{i+1}(t)\right)$ to the set $\{(0,0,1),(1,0,1)\}$ given that the chain starts in $\{(0,1,0),(0,1,1)\}$ at initial time. By analogy with the derivation of $\tilde{\Xi}_{i}(s)$, assuming that $\{(0,0,1),(1,0,1)\}$ is an absorbing set, $\tilde{\Xi}_{i}^{*}(s)$ becomes the LST of the time to absorption of $\left(L_{i-1}(t), L_{i}(t), L_{i+1}(t)\right)$ with $\mathbf{A}+\mathbf{B}$, the transient states generator, $u$ transition rate column vector from transient states to the absorbing set, and with initial probability distribution $y^{*}$. That is, the probability distribution that the chain starts in $\{(0,1,0),(0,1,1)\}$ is given by

$$
y^{*}=\frac{\left(0, \alpha_{i+1}^{1}, \alpha_{i+1}^{0}, 0,0,0\right)}{\alpha_{i+1}^{0}+\alpha_{i+1}^{1}} .
$$

Lemma 6.(b) gives the desired result of $\tilde{\Xi}_{i}^{*}(s)$. 